\title{
Spherically symmetric anisotropic charged solution under complete geometric deformation approach
}

\author{
S. K. Maurya ${ }^{\mathrm{a}}$, Asma Mohammed Al Aamri ${ }^{\mathrm{b}}$, Athari Khalifa Al Aamri ${ }^{\mathrm{c}}$, Riju Nag ${ }^{\mathrm{d}}$ \\ Department of Mathematical and Physical Sciences, College of Arts and Science, University of Nizwa, Nizwa, Sultanate of Oman
}

Received: 21 June 2021 / Accepted: 25 July 2021 / Published online: 6 August 2021

(C) The Author(s) 2021

\begin{abstract}
We present a new systematic approach to find the exact gravitationally decoupled anisotropic spherical solution in the presence of electric charge by using the complete geometric deformation (CGD) methodology. To do this, we apply the transformations over both gravitational potentials by introducing two unknown deformation functions. This new systematic approach allows us to obtain the exact solution of the field equations without imposing any particular ansatz for the deformation functions. Specifically, a wellknown mimic approach and equation of state (EOS) have been applied together for solving the system of equations, which determine the radial and temporal deformation functions, respectively. The matching conditions at the boundary of the stellar objects with the exterior Reissner-Nordström metric are discussed in detail. In order to see the physical validity of the solution, we used well-behaved interior seed spacetime geometry and solved the system of equations using the above approaches. Next, we presented several physical properties of the solution through their graphical representations. The stability and dynamical equilibrium of the solution have been also discussed. Finally, we predicted the radii and mass-radius ratio for several compact objects for different decoupling parameters together with the impact of the decoupling parameters on the thermodynamical observables.
\end{abstract}

\section{Introduction}

As the compact stars are the final stages of the evolution of an ordinary star, they provide great testing conditions for studying tremendously dense objects in extreme conditions. In the recent past, various compact objects have been observed in

\footnotetext{
a e-mail: sunil@unizwa.edu.om (corresponding author)

be-mail: 14219912@uofn.edu.om

c e-mail: 09268242@uofn.edu.om

de-mail: rijunag@gmail.com
}

the form of pulsars and spinning stars having a strong magnetic field [1]. The basic theoretical foundation to understand the compact stars have been based on Fermi Dirac statistics, which explains the pressure degeneracy in the extreme condition that saves the compact stars from gravitational collapse [2]. Later, by combining Einstein's special theory of relativity and various principles of quantum physics, the white dwarfs, which were supported solely by degenerate gas of electrons, were recognized as compact stars by Chandrasekhar $[3,4]$. It was shown, that the white dwarfs can have a maximum size of about 1.4 times the mass of the sun or approximately $3 \times 10^{30} \mathrm{~kg}$.

As of now, there is no complete theory that explains extremely dense matter with a highly interactive environment. One probable explanation can be, that such dense nuclear matter does not only contain the nucleons and leptons but can also include many exotic particles in their different forms such as different mesons, hyperons, baryon resonances, strange quark matter, etc. So the exact composition of such compact stellar objects needs much deeper research. This is why the structural aspects and formation of these extremely dense stellar objects have been still an active research field.

The solution to Einstein's field equations (EFE) unlocks many mysteries of the universe. But, due to its non-linearity, it is a great challenge to find exact solutions to Einstein's field equations (EFE). Although throughout the last century, many exact solutions of EEE have been found, but amongst them very few can describe realistic astrophysical compact objects. As a result, till the present date, the researchers are trying to find the exact solutions of EFE in several contexts. Mainly two methodologies are taken into account to solve the system, one is to decrease energy density or consider a metric function. This approach leads to other unknown functions that can be found by imposing the pressure isotropy conditions. But the drawback of this approach is that it doesn't always provide an acceptable exact solution. Another way 
one can solve EFE is by considering an equation of state (EoS) that relates the pressure and density. But yet, because of complex integral forms, this way also wasn't efficient in finding exact solutions of EFE. However, it was shown, when the matter density is more than nuclear density, i.e. very high, then the matter distribution is not necessarily isotropic, and there may be the presence of anisotropic pressure $\left(p_{r} \neq p_{t}\right)$ [5-10]. So if we consider anisotropic fluid, the EFE in presence of anisotropy can be easily solved in contrast to isotropic fluid distribution.

To consider the real situation going on inside a compact object, the function called anisotropic factor has great use. As suggested by Rago [11] we can use two different functions in order to get the solution of field equations in anisotropic model. They are, the anisotropic factor $\left(\Delta=p_{r}-p_{t}\right)$ and the generating function. This anisotropic factor comes as pressure splits into two components in extreme conditions, i.e. radial and tangential pressure components. It is evident from the expression of the anisotropic factor $\Delta$ that its sign can be either positive or negative. Positive $\Delta$ signifies that the force is acting outwards as the tangential pressure dominates the radial pressure, whereas the negative $\Delta$ implies inward force as here the radial pressure dominates the tangential pressure.

The anisotropy can be originated from various things such as extremely high density in the core, a mixture of different types of fluids, presence of the magnetic field, rotational motion, various condensate states (such as meson condensates, pion condensates, etc.), superfluid 3A, etc. As mentioned by Bowlers and Liang [12], no celestial object can have a perfectly isotropic matter distribution, and specifically for compact objects, the anisotropy is more evident due to extreme gravitational pull and density. The concept of fluid pressure being anisotropic was first proposed by Ruderman [5] and after that by Canuto and Chitre [6]. Various aspects of anisotropy were discussed by Herrera and Santos in a review paper [13]. It is important to mention that in the context of compact stars, the chances of anisotropy are much higher because of the relativistic nature of the interaction between the particles. Due to this, the motion of the particles becomes too random to maintain the isotropy for any specific region. Also, the anisotropic force affects the density of the star and due to this, the density of the compact star becomes different in different regions of the star. So the real nature of the compact star becomes anisotropic in nature with variable density. In the context of anisotropy, various diversified works can be found in the following references [14-26].

Many researchers are using various modified theories of gravity in various ways to explain realistic compact objects in the recent time. These modified theories of gravity coupled with several other approaches often have been able to describe many exotic features of compact stars which otherwise was a challenge. These days, a specific approach namely gravitational decoupling using minimal geometric deformation (MGD) has attracted a lot of attention amongst researchers. It was first discovered by Ovalle $[27,28]$ and was used in the framework of Randall-Sundrum brane-world [29, 30] with the purpose of deforming the Schwarzschild spacetime. Contreras and his collaborators $[31,32]$ investigated the technique to determine anisotropic solution from any isotropic solution and vice versa using MGD in the context of black holes. By using MGD, a few well-recognized Einstein field solutions were studied for the isotropic, anisotropic, and charged cases [33-59]. Recently, Contreras et al. [60] has also introduced the MGD in axially symmetric systems and rotating black holes. Some other works on dark matter, black holes and Dirac stars in the context of MGD can be also seen in the following Refs. [61-64]. In this connection, it is important to mention that in addition to being a new effective tool, the MGD and extended MGD [called complete geometric deformation (CGD)] approaches are also efficient in finding the new solutions of the Einstein's field equations. These approaches can be executed in two opposite ways: the first one, If we solve to Einstein's field equations for a very complicated energy-momentum tensor $\tilde{T}_{i j}$, then we simply split this complicated $\tilde{T}_{i j}$ into two relatively simple energymomentum tensor components such as: $\tilde{T}_{i j}=\left\{T_{i j}, \theta_{i j}^{n}\right\}$.

Then, we solve Einstein's field equations together with each of the split components and obtain various solutions corresponding to each separate components for the main energy-momentum tensor $\tilde{T}_{i j}$. Finally, the linear combination of each solution obtained for the components is taken as the solution for the original energy-momentum tensor. while, the second approach is exactly opposite to the aforementioned earlier approach. In the approach, a relatively simple energy-momentum tensor is converted to a complicated one by adding an extra source into the original energymomentum tensor to generalize a simple solution of Einstein's field equations. This can be done in the following way: $T_{i j} \mapsto \tilde{T}_{i j}=T_{i j}+\theta_{i j}^{1}$.

One can repeat this process by adding more sources $\left(\theta_{i j}^{n}\right)$ to generalize the solution even more as necessary.

On the other hand, there is another approach to use gravitational decoupling and that is to modify the Einstein-Hilbert (EH) action by adding an extra Lagrangian density corresponding to an extra source such as, $S=S_{E H}+S_{\theta}$, where $S_{E H}$ representing EH action while $S_{\theta}$ is the action corresponding to extra source. By varying the aforementioned action $S$ with respect to the metric tensor $g^{i j}$ one obtains the general equation of motion for the total energy momentum tensor $\left(T_{i j}^{\text {tot }}\right)$ which is a combination of $T_{i j}$ and the additional term $\theta_{i j}$ such that $T_{i j}^{\mathrm{tot}}=T_{i j}+\theta_{i j}$, where $T_{i j}$ is either perfect fluid or anisotropic fluid matter distribution and $\theta_{i j}$ represents an extra source. It is important to mention that the MGD transformation is a particular transformation that works only along the radial metric component, while the temporal part 
remains unchanged. Many physically acceptable solutions for spherically symmetric space-time were found using this transformation in various contexts including modified gravity theory [65-71]. Also, this approach of MGD has proven effective beyond the GR while examining the gravitational lensing singularity [72].

But later on, Ovalle along with his collaborators suggested that this approach of considering only radial transformation has its drawbacks, such as it can't explain the presence of a stable black hole with a horizon that is well defined. In this regard the MGD was extended by them by considering the deformation along both radial and temporal metric functions. Using this extended gravitational decoupling (EGD) approach, they developed a modified Schwarzschild geometry and find a new solution that describes the well-behaved brane-world star [73]. Mathematically speaking, in extended MGD, the deformation of MGD extends in the following way [74]:

$\nu(r) \mapsto \xi(r)+\alpha h(r), \quad$ and $\quad \lambda(r) \mapsto-\ln [\mu(r)+\alpha f(r)]$.

Despite the appearance of a new degree of freedom $h(r)$ in the $\theta$-sector, the problem can still be solved mathematically. There can be many ways to close this $\theta$-sector appearing this extra degree of freedom $h(r)$ such as: using embedding class I methodology or applying the mimic approach etc. together with considering a particular well-behaved ansatz for deformation, etc. It is important to note that the entire hydrostatic balance gets modified due to the deformation of both metric functions. This is a significant point as the fulfillment of the conservation law of energy-momentum tensor depends heavily on the hydrostatic balance. So while working with EGD, it is very important to check the hydrostatic balance to find out the model is acceptable realistically or not. In light of EGD, some recent research work can be found in references [75,76].

But in the present article, we have applied the extended gravitational decoupling by means of the complete geometric deformation approach for solving the Einstein-Maxwell's field equations without considering any ansatz for the deformation function. The initial system has been solved by taking Tolman-Kuchowicz spacetime geometry for the perfect fluid charged matter distribution. The field equations corresponding to $\theta$-sector, which depend on mainly two unknowns (called deformation functions $f(r)$ and $h(r)$ ), have been solved by two different approaches. In particular, the deformation function $f(r)$ is determined by mimic approach: $\rho(r)=\theta_{0}^{0}(r)$ while $h(r)$ has been obtained through the equation of state (EOS): $\theta_{1}^{1}=\beta \theta_{0}^{0}+\gamma$, where $\beta$ and $\gamma$ are constants. We would like to mention here that this approach has been applied first time to obtain both the deformation functions, and solved exactly the system of equations corresponding to the $\theta$-sector.
The present article is organized as follows: In Sect. 2, we discussed the modified action and decoupled fields equations under the extended gravitational decoupling system which has divided into three Sects. 2.1, 2.2, and 2.3. In Sect. 2.1, we discussed the modified action for the Einstein-Maxwell system, while the Einstein-Maxwell's field for the decoupled system were explained in Sect. 2.2, and the Sect. 2.3 includes the procedure to split the decoupled field equations under extended gravitational decoupling. In Sect. 3, we obtained an extended gravitationally decoupled solution, which was divided into two Sects. 3.1 and 3.2. In Sect. 3.1, the mimicking approach for determining $f(r)$ is discussed while the equation of state approach has been mentioned in Sect. 3.2, which determines the function $h(r)$. A detailed discussion about the boundary conditions have been mentioned in Sect. 4, while in Sect. 5, we shed light on the regular behavior of deformed gravitational potential, together with deformation functions. The Sect. 6 contains the discussion for the physical properties of the model which is divided into four subsections: In Sect. 6.1, the physical behavior of total radial and tangential pressure, total energy density, and total anisotropy were discussed, however the energy conditions are analyzed in Sect. 6.2. In Sect. 6.3, the compactness relation and surface gravitational redshift are explained while the stability and dynamical equilibrium are discussed in Sect. 6.4. The impact of the coupling constant $\alpha$ on the thermodynamic observables is explained in Sect. 7. The final section is kept for the conclusion and a brief discussion about the results.

\section{Modified action and decoupled field equations under extended gravitational decoupling system}

2.1 The modified action for the Einstein-Maxwell's system

With the inclusion of an extra Lagrangian density for an extra source, the modified action for gravitational decoupled systems can be defined as [74],

$S=S_{E H}+\alpha S_{\theta}=\int\left[\frac{R}{16 \pi}+\mathcal{L}_{\mathcal{M}}+\mathcal{L}_{e}+\alpha \mathcal{L}_{\theta}\right] \sqrt{-g} d^{4} x$.

With the symbols $g$ and $R$ having their usual meanings. The Lagrangian for the matter field is denoted by $\mathcal{L}_{\mathcal{M}}$ and Lagrangian for electric field is denoted as $\mathcal{L}_{e}$. Also, the Lagrangian density due to an additional source is described as $\mathcal{L}_{\theta}$. Now, for the matter field $\mathcal{L}_{M}$, the energy-momentum tensor $T_{i j}$ is defined as,

$T_{i j}=\frac{2}{\sqrt{-g}} \frac{\delta\left(\sqrt{-g} \mathcal{L}_{M}\right)}{\delta g^{i j}}$. 
As it is known that matter Lagrangian $\mathcal{L}_{\mathcal{M}}$ is only dependent on metric tensor $g_{i j}$ components, while being independent of their derivatives, which is obtained as

$T_{i j}=\frac{2 \partial\left(\mathcal{L}_{\mathcal{M}}\right)}{\partial g^{i j}}-g_{i j} \mathcal{L}_{\mathcal{M}}$

Also, corresponding to the Lagrangian density $\mathcal{L}_{e}$ and $\mathcal{L}_{\theta}$ the electromagnetic field tensor $E_{i j}$ and the extra source $\theta_{i j}$ are given by,

$E_{i j}=-g_{i j} \mathcal{L}_{e}+2 \frac{\partial \mathcal{L}_{e}}{\partial g^{i j}}$

$\theta_{i j}=2 \frac{\delta \mathcal{L}_{\theta}}{\delta g^{i j}}-g_{i j} \mathcal{L}_{\theta}$

Now, the following general equations of motion due to the charged, decoupled system can be obtained by the variation of action (1) with respect to the metric tensor $g^{i j}$ as,

$G_{i j}=-8 \pi\left(T_{i j}^{\mathrm{tot}}+E_{i j}\right)$

where,

$T_{i j}^{\mathrm{tot}}=T_{i j}+\alpha \theta_{i j}$

Here relativistic units are considered with $G=c=1$, and Einstein tensor is expressed as $G_{i j}$. The $E_{i j}$ and $T_{i j}$ corresponding perfect fluid distribution are defined as,

$$
\begin{aligned}
E_{i j} & =\frac{1}{4 \pi}\left(-F_{i}^{m} F_{j m}+\frac{1}{4} g_{i j} F_{\gamma n} F^{\gamma n}\right) \\
T_{i j} & =(\rho+p) u_{i} u_{j}-p g_{i j} .
\end{aligned}
$$

Here matter density is denoted by $\rho$ while pressure is represented as $p$. Also, $u^{i}$ being a covariant component of the four velocity, fulfills $u_{i} u^{j}=1$ and $u^{i} \nabla^{i} u_{i}=0$ respectively. Moreover, the Maxwell's field equations are satisfied by the electromagnetic field tensor $F_{i j}$ as,

$$
\nabla_{j}\left[(-g)^{1 / 2} F^{i j}\right]=\frac{4 \pi}{(-g)^{-\frac{1}{2}}} j^{i} \text { and } F_{i j, \gamma}+F_{j \gamma, i}+F_{\gamma i, j}=0 .
$$

where, electromagnetic four current vector $j^{i}$ is given as,

$J^{i}=\frac{\sigma}{\sqrt{g 44}} \frac{d x^{i}}{d x^{4}}=\sigma v^{i}$

Furthermore, the interior space-time of the stellar object is considered to be a static and spherically symmetric which can be described by the following line element,

$d s^{2}=-e^{\lambda(r)} d r^{2}-r^{2}\left(d \theta^{2}+\sin ^{2} \theta d \phi^{2}\right)+e^{\nu(r)} d t^{2}$,

where, the metric potentials $v$ and $\lambda$ are only radially dependent. Then, the charge density $\sigma$ for the line element (12) is defined as $\sigma=e^{v / 2} J^{0}(r)$. Here, $J^{0}$ is a non-vanishing component of the four-current for static fluid matter distribution which leads the four-current to act only radially because of the spherical symmetry. So, the corresponding non-zero components of electromagnetic field tensor $F^{01}$ and $F^{10}$ are related as $F^{01}=-F^{10}$. Thus, $F^{01}$ and $F^{10}$ can be enough to define the radial electric field. Then the non-vanishing and anti-symmetric electric field components $F^{01}$ and $F^{10}$ for the spherically symmetric line element (12) in terms of electric charge can be obtained as,

$F^{01}=-F^{10}=\frac{q}{r^{2}} e^{-(\nu+\lambda) / 2}$

where the electric charge within the compact star of radius $r$ is denoted by $q(r)$. The electric field $E$ and electric charge $q(r)$ can be defined using relativistic Gauss Law as,

$$
\begin{aligned}
& E^{2}=-F_{10} F^{10}=\frac{q^{2}}{r^{4}} . \\
& q(r)=4 \pi \int_{0}^{r} \sigma r^{2} e^{\lambda / 2} d r=r^{2} \sqrt{-F_{10} F^{10}},
\end{aligned}
$$

Now, the components for $T_{i}^{\mathrm{j}}$ and $E_{i}^{\mathrm{j}}$ can be written as,

$$
\begin{aligned}
& T_{0}^{0}=\rho(r), T_{1}^{1}=-p(r), T_{2}^{2}=-p(r), \\
& E_{0}^{0}=E_{1}^{1}=-E_{2}^{2}=\frac{1}{8 \pi} \frac{q^{2}(r)}{r^{4}} .
\end{aligned}
$$

It is important to note that the interior matter distribution becomes anisotropic with the inclusion of new source $\theta_{i j}$ for the condition $\theta_{2}^{2} \neq \theta_{1}^{1}$. In this case, the total energy momentum tensor $T_{i j}^{\text {tot }}$ will represent an anisotropic matter distribution. Hence, the components of $T_{i j}^{\text {tot }}$ can be given as,

$\left(T_{0}^{0}\right)^{\text {tot }}=\rho^{\text {tot }},\left(T_{1}^{1}\right)^{\text {tot }}=-p_{r}^{\text {tot }}$, and $\left(T_{2}^{2}\right)^{\text {tot }}=-p_{t}^{\text {tot }}$.

and then,

$\rho^{\text {tot }}=\rho+\alpha \theta_{0}^{0}, p_{r}^{\text {tot }}=p-\alpha \theta_{1}^{1}$, and $p_{t}^{\text {tot }}=p-\alpha \theta_{2}^{2}$.

On the other hand, the anisotropic factor can be provided as follows,

$$
\Delta(r)=p_{t}^{\mathrm{tot}}(r)-p_{r}^{\mathrm{tot}}(r)=\alpha\left[\theta_{1}^{1}(r)-\theta_{2}^{2}(r)\right] .
$$

Now we move on to the derivation of the Einstein field equations for decoupled system as:

\subsection{Einstein-Maxwell's field for decoupled system}

Under the spherically symmetric static spacetime (12), the Einstein-Maxwell's field equations corresponding to decoupling system (6) can be written as,

$$
\begin{aligned}
& \rho^{\text {tot }}+\frac{q^{2}}{8 \pi r^{4}}=\frac{e^{-\lambda}}{8 \pi}\left(\frac{\lambda^{\prime}}{r}-\frac{1}{r^{2}}+\frac{e^{\lambda}}{r^{2}}\right), \\
& p_{r}^{\text {tot }}-\frac{q^{2}}{8 \pi r^{4}}=\frac{e^{-\lambda}}{8 \pi}\left(\frac{\nu^{\prime}}{r}+\frac{1}{r^{2}}-\frac{e^{\lambda}}{r^{2}}\right),
\end{aligned}
$$




$$
p_{t}^{\text {tot }}+\frac{q^{2}}{8 \pi r^{4}}=\frac{e^{-\lambda}}{32 \pi}\left(2 v^{\prime \prime}+v^{\prime 2}+2 \frac{v^{\prime}-\lambda^{\prime}}{r}-v^{\prime} \lambda^{\prime}\right) .
$$

Thus, we can get the following conservation equation from the linear combination of the Eqs. (21)-(23) as,

$\left(p_{r}^{\mathrm{tot}}\right)^{\prime}+\frac{v^{\prime}}{2}\left(\rho^{\mathrm{tot}}+p_{r}^{\mathrm{tot}}\right)-\frac{q q^{\prime}}{4 \pi r^{4}}+\frac{2}{r}\left(p_{r}^{\mathrm{tot}}-p_{t}^{\mathrm{tot}}\right)=0$.

and corresponding mass function of the object can be determined by the formula,

$$
\begin{aligned}
m(r) & =4 \pi \int_{0}^{r} x^{2} \rho^{\mathrm{tot}}(x) d x+\frac{1}{2} \int_{0}^{r} \frac{q^{2}(x)}{x^{2}} d r+\frac{q^{2}(r)}{2 r} \\
& =\frac{r}{2}\left[1-e^{-\lambda(r)}+\frac{q^{2}(r)}{r^{2}}\right] .
\end{aligned}
$$

2.3 The procedure to split the decoupled field equations under extended gravitational decoupling for the sources $T_{i j}$ and $\theta_{i j}$

The procedure for splitting of the decoupled field equations (21)-(23) by the extended gravitational decoupling in two separate systems (Einstein-Maxwell system and quasiEinstein system) is discussed in this section. Here, energy tensor $T_{i j}$ is considered for the Einstein-Maxwell system having perfect fluid matter distribution while quasi-Einstein system is taken for the extra source $\theta_{i j}$. Now, the Ovalle [74] transformation in metric potentials is applied as,

$$
\begin{aligned}
& \xi \mapsto v=\xi+\alpha h(r) \\
& \mu \mapsto e^{-\lambda}=\mu+\alpha f(r),
\end{aligned}
$$

where $h(r)$ denotes the geometric deformation function for the temporal metric component and $f(r)$ corresponds to the radial metric component. It is noted that the coupling constant $\alpha$ is a real number. Also, it is important to note that the above transformation is known as complete geometric deformation (CGD) that can be also called an extended case of minimal geometric deformation (MGD) means transformations along both radial and temporal components of the line element. With the substitution of the metric functions (26) and (27) with Eq. (19) in the field equations of decoupled systems (21)-(23), the following set of equations are being arrived:

First one corresponding to $\alpha=0$, for the charged perfect fluid distribution, the Einstein-Maxwell field equations are given as,

$$
\begin{aligned}
& 8 \pi \rho+\frac{q^{2}}{r^{4}}=\frac{1-\mu}{r^{2}}-\frac{\mu^{\prime}}{r} \\
& 8 \pi p-\frac{q^{2}}{r^{4}}=\frac{\mu-1}{r^{2}}+\frac{\mu \xi^{\prime}}{r}, \\
& 8 \pi p+\frac{q^{2}}{r^{4}}=\mu\left(\frac{\xi^{\prime \prime}}{2}+\frac{\xi^{\prime 2}}{4}+\frac{\xi^{\prime}}{2 r}\right)+\left(\frac{\xi^{\prime} \mu^{\prime}}{4}+\frac{\mu^{\prime}}{2 r}\right)
\end{aligned}
$$

along the conservation equation,

$$
p^{\prime}+\frac{\xi^{\prime}}{2}(\rho+p)-\frac{q q^{\prime}}{4 \pi r^{4}}=0 .
$$

The internal structure of the compact object with charged perfect fluid matter distribution can be obtained from the conservation equation (31), which is satisfied by the solution of the field equations (28)-(30). The following line element provides the corresponding solution,

$d s^{2}=-\mu d r^{2}-r^{2}\left(d \theta^{2}+\sin ^{2} \theta d \phi^{2}\right)+e^{\xi} d t^{2}$

where $m_{0}$ is the mass function for charged matter for the standard GR expressions (28)-(30), which can be given as,

$$
\frac{2 m_{0}}{r} \equiv 1-\mu+\frac{q^{2}}{r^{2}} \equiv \frac{8 \pi}{r} \int_{0}^{\mathrm{r}}\left[\rho(x)+\frac{q^{2}(x)}{8 \pi x^{2}}\right] x^{2} d x+\frac{q^{2}}{r^{2}} .
$$

Now let's focus on the parameter $\alpha$ for observing the effects on the charged perfect fluid solution $\xi, \mu \rho, p$ coming from the extra source $\theta_{i j}$. For this reason, the field equations associated with the extra source $\theta_{i j}$ for the quasi-Einstein system can be written as,

$$
\begin{aligned}
8 \pi \theta_{0}^{0}= & -\left(\frac{f^{\prime}}{r}+\frac{f}{r^{2}}\right), \\
8 \pi \theta_{1}^{1}= & -f\left(\frac{v^{\prime}}{r}+\frac{1}{r^{2}}\right)-\frac{\mu h^{\prime}}{r}, \\
8 \pi \theta_{2}^{2}= & -\frac{f}{2}\left(v^{\prime \prime}+\frac{v^{\prime 2}}{2}+\frac{v^{\prime}}{r}\right)-\frac{\alpha f^{\prime}}{2}\left(\frac{v^{\prime}}{2}+\frac{1}{r}\right)-\frac{\mu^{\prime} h^{\prime}}{4} \\
& -\frac{\mu}{4}\left(2 h^{\prime \prime}+\alpha h^{\prime 2}+\frac{2 h^{\prime}}{r}+2 \xi^{\prime} h^{\prime}\right) .
\end{aligned}
$$

Also, the following conservation equation can be obtained from the linear combination of quasi-Einstein equations (34)-(36) as,

$$
-\frac{v^{\prime}}{2}\left(\theta_{0}^{0}-\theta_{1}^{1}\right)+\left(\theta_{1}^{1}\right)^{\prime}-\frac{2}{r}\left(\theta_{2}^{2}-\theta_{1}^{1}\right)=\frac{h^{\prime}}{2}(p+\rho) .
$$

It can be clearly understood that the extra source $\theta_{i j}$ results in the anisotropy inside the compact structure (Fig. 1).

\section{Extended gravitationally decoupled solution}

In this section, we will determine the solutions for both systems Eqs. (28)-(30) and Eqs. (34)-(36) under the complete geometric deformation approach known as the extended gravitational decoupling approach. To do this, first we find the solution of seed system for charged perfect fluid solution which depends on five unknowns $\{\mu, \xi, \rho, p, q\}$. To solve the seed system, we take well-behaved interior space-time as, 


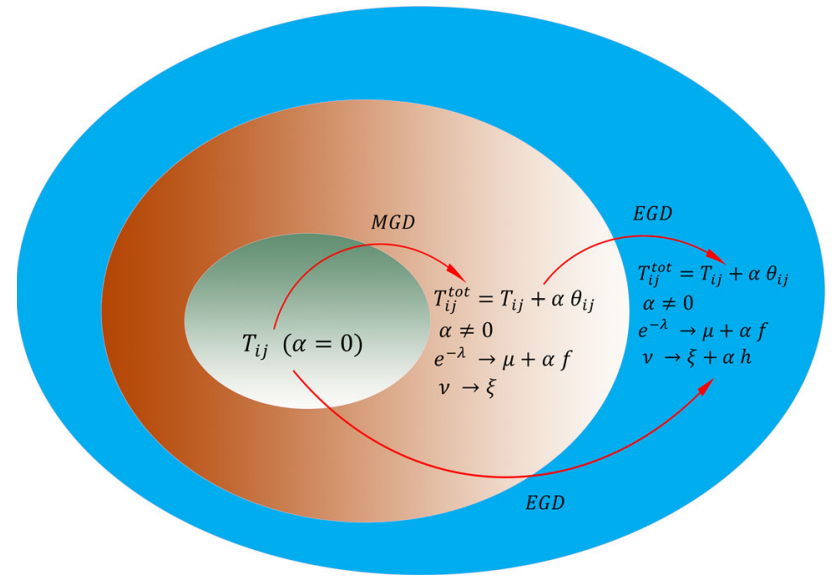

Fig. 1 This diagram shows that how any perfect fluid matter distribution can be consistently extended into the anisotropic domain though the MGD-decoupling as well as CGD-decoupling approach

$d s^{2}=-\left(1+a r^{2}+b r^{4}\right) d r^{2}-r^{2}\left(d \theta^{2}+\sin ^{2} \theta d \phi^{2}\right)+e^{B r^{2}+C} d t^{2}$,

where $B, a$ and $b$ are constants with units $l^{-2}, l^{-2}$ and $l^{-4}$ and $C$ is a dimensionless constant. Then the seed geometry $\mu$ and $\xi$ can given as: $\mu=1 /\left(1+a r^{2}+b r^{4}\right)$ and $\xi=B r^{2}+C$. Now by plugging of $\xi$ and $\mu$ into Eqs. (28)-(30), we obtain the expressions for electric charge $(q)$, pressure $(p)$, and density $(\rho)$ as,

$$
\begin{aligned}
\frac{q^{2}}{r^{4}}= & \frac{1}{2\left(1+a r^{2}+b r^{4}\right)^{2}}\left[r ^ { 2 } \left(a^{2}+B^{2}+b^{2} r^{4}+a(-B\right.\right. \\
& \left.\left.\left.+2 b r^{2}+B^{2} r^{2}\right)+b\left(-1-2 B r^{2}+B^{2} r^{4}\right)\right)\right], \\
8 \pi p= & \frac{1}{2\left(1+a r^{2}+b r^{4}\right)^{2}}\left[2 B\left(2+b r^{4}\right)-a^{2} r^{2}-b r^{2}\left(3+b r^{4}\right)\right. \\
& \left.+a\left(-2+3 B r^{2}-2 b r^{4}+B^{2} r^{4}\right)+B^{2}\left(r^{2}+b r^{6}\right)\right], \\
8 \pi \rho= & \frac{1}{2\left(1+a r^{2}+b r^{4}\right)^{2}}\left[a^{2} r^{2}+a\left(6+B r^{2}+2 b r^{4}-B^{2} r^{4}\right)\right. \\
& \left.+r^{2}\left(-B^{2}+b^{2} r^{4}+b\left(11+2 B r^{2}-B^{2} r^{4}\right)\right)\right] .
\end{aligned}
$$

Since we have already specified the complete geometry of the seed system which was required for obtaining the solution for another system, known as quasi-Einstein system, (34)-(36). As we see that this system mainly depends on two unknowns $f(r)$ and $h(r)$, called deformation functions. So, we will adopt two different approaches for determining these deformation functions $f(r)$ and $h(r)$. The function $f(r)$ is determined by using a well-known constraint procedure, specifically mimicking of the density constraints i.e. $\rho(r)=\theta_{0}^{0}(r)$, which is mentioned in the next section:
3.1 Mimicking to the density constraints $\left[\rho(r)=\theta_{0}^{0}(r)\right]$ approach for determining $f(r)$

From Eqs. (28) and (34), the mimicking to the density constraints $\left[\rho(r)=\theta_{0}^{0}(r)\right]$ procedure provides a differential equation in $f(r)$ of the form,

$f^{\prime}+\frac{1}{r} f+\frac{r\left[a\left(6+B r^{2}+2 b r^{4}-B^{2} r^{4}\right)+g(r)\right]}{2\left(1+a r^{2}+b r^{4}\right)^{2}}=0$,

where, $g(r)=a^{2} r^{2}+r^{2}\left[-B^{2}+b^{2} r^{4}+b\left(11+2 B r^{2}-\right.\right.$ $\left.\left.B^{2} r^{4}\right)\right]$. On integrating of the above differential which leads an expression for deformation function $f(r)$ as,

$$
\begin{aligned}
f= & {\left[\left(\frac{\sqrt{2} f_{2}}{8 r f_{4} f_{5} b^{3 / 2}}-\frac{\sqrt{2} f_{1}}{8 r f_{4} f_{5} b^{3 / 2}}\right) \tan ^{-1}\left(\frac{\sqrt{2 b} r}{f_{5}}\right)\right.} \\
& \left.+\frac{\left(B^{2}-b\right)}{2 b}+\frac{2\left(5+B r^{2}\right)}{4\left(1+a r^{2}+b r^{4}\right)}\right] .
\end{aligned}
$$

We would like to mention that all the calculations have been done by taking the following assumption to avoid the singularity in the expression of $f(r)$ as: (i): By expanding the $\tan ^{-1}(x)$, where $x=\sqrt{2 b} r / f_{5}$, up to linear term using Taylor series expansion around $x=0$, (ii) The integration constant involved in the solution is taken to be zero. ${ }^{1}$ Now, the other deformation function $h(r)$ will be obtained by taking a linear equation of state (EOS) between the $\theta$-components which we discuss in the next section as:

\subsection{Equation of state approach for determining $h(r)$}

We use the following EOS in $\theta$-components to determine the function $h(r)$ as:

$\theta_{1}^{1}=\beta \theta_{0}^{0}+\gamma$

where $\beta$ and $\gamma$ are the constants. This EOS will provide a first order linear differential equation, whose solution yields the following expression for $h(r)$ as:

$$
\begin{aligned}
h= & \frac{1}{100}\left[-h_{1}(r)-h_{2}(r)+\frac{h_{11}(r) h_{6}(r)}{h_{7}(r)}-\frac{20(5 B-2 \gamma) r^{2}}{\alpha}\right. \\
& \left.+\frac{h_{12}(r)\left[h_{8}(r)+b B\left(h_{9}(r)-B h_{10}(r) \sqrt{\alpha}\right) \sqrt{\alpha}\right]}{b \alpha^{3 / 2} A_{3}\left[b A_{2}+B \alpha\left(B \alpha-a A_{2}\right)\right]}\right] .
\end{aligned}
$$

Now we have solved completely the field equations for $\theta$ sector, then the expressions for $\theta_{0}^{0}, \theta_{1}^{1}$ and $\theta_{2}^{2}$ are given as, ${ }^{2}$

$$
8 \pi \theta_{0}^{0}(r)=\frac{a^{2} r^{2}+a F_{1}(r)+r^{2}\left[-B^{2}+b^{2} r^{4}+b F_{2}(r)\right]}{2\left(1+a r^{2}+b r^{4}\right)^{2}},
$$

\footnotetext{
$\overline{1}$ The expressions for unknown functions involved in the $f(r)$ are mentioned in the Appendix.

2 The expressions for unknown functions involve in the $\theta$-sector components are mentioned in the Appendix.
} 


$$
\begin{aligned}
& 8 \pi \theta_{1}^{1}(r)=\frac{\left.F_{3}(r)+5 b^{2} \beta r^{6}+4 b^{2} \gamma r^{8}+B \beta F_{4}(r)+\theta_{11}(r)\right]}{4\left(1+a r^{2}+b r^{4}\right)^{2}}, \\
& 8 \pi \theta_{2}^{2}(r)=\frac{\left[\theta_{21}(r)+\theta_{22}(r)+a^{2} r^{2}\left(\theta_{23}(r)+\theta_{24}(r)\right]\right.}{16\left(1+a r^{2}+b r^{4}\right)^{3}\left(5 a \alpha r^{2}-B \alpha r^{2}+5 b \alpha r^{4}-4\right)} .
\end{aligned}
$$

Then the deformed gravitational potentials can be written as:

$$
e^{\lambda(r)}=\frac{1+a r^{2}+b r^{4}}{1+\alpha\left(1+a r^{2}+b r^{4}\right) f(r)}, \quad e^{\nu(r)}=e^{B r^{2}+C+\alpha h(r)}
$$

\section{Matching conditions}

To study the stellar distribution, the matching condition is a pivotal part between the interior $(r<R)$ and exterior $(r>R)$ of the star at the surface $(r=R)$. The interior stellar geometry is provided by the extended geometric deformation line element in the current study as,

$$
\begin{aligned}
d s^{2}= & -\left(1-\frac{2 m(r)}{r}+\frac{q^{2}}{r^{2}}\right)^{-1} d r^{2}-r^{2}\left(d \theta^{2}-\sin ^{2} \theta d \phi^{2}\right) \\
& +e^{\xi(r)+\alpha h(r)} d t^{2}
\end{aligned}
$$

where, internal mass of the stellar structure for total energy tensor $T_{i j}^{\text {tot }}$ is given by $m(r) .{ }^{3}$ Now, the interior space-time geometry (50) must be smoothly matched with the suitable exterior (vacuum) space-time at the boundary of the star $(r=$ $R$ ). In this context, as proposed by Ovalle [74] for "Maxwell version" of the vacuum solution $T_{i j}=0$, the exterior spacetime for extended gravitational decoupling can be given by a well-known Reissner-Nordström solution as,

$$
\begin{aligned}
d s^{2}= & -\left(1-\frac{2 M}{r}+\frac{Q^{2}}{r^{2}}\right)^{-1} d r^{2}-r^{2}\left(d \theta^{2}-\sin ^{2} \theta d \phi^{2}\right) \\
& +\left(1-\frac{2 M}{r}+\frac{Q^{2}}{r^{2}}\right) d t^{2}
\end{aligned}
$$

where total charge and total mass of the compact objects of radius $R$ are $q(R)=Q$ and $m(R)=M$, respectively. To match the inner manifold with the external manifold at the boundary, the Israel-Darmois junction conditions procedure $[77,78]$ is applied for smooth joining. The method to join both space-time at the boundary across the surface $\sum$ is called the continuity of first and second fundamental forms. According to the first fundamental form, the intrinsic geometry $g_{i j}$ induced by $\mathcal{M}^{-}$and $\mathcal{M}^{+}$on the interface meets

$g_{t t}^{-}\left|r=R=g_{t t}^{+}\right| r=R, \quad g_{r r}^{-}\left|r=R=g_{r r}^{+}\right| r=R$.

$\overline{{ }^{3} \text { Where } m(r)}=m_{0}(r)-\frac{\alpha r}{2} f(r)$ and $m_{0}(R)=M_{0}$.
By expressing the first fundamental form explicitly as,

$$
\begin{aligned}
& e^{-\lambda(R)}=\left(1-\frac{2 M}{R}+\frac{Q^{2}}{R^{2}}\right), \\
& e^{\nu(R)}=\left(1-\frac{2 M}{R}+\frac{Q^{2}}{R^{2}}\right),
\end{aligned}
$$

while the continuity of the second fundamental form gives,

$$
T_{i j}^{\mathrm{tot}}=0 \Longrightarrow\left[p_{r}^{\mathrm{tot}}\right]_{\Sigma}=\left[p-\theta_{1}^{1}(r)\right]_{\Sigma}=0 .
$$

Now, the vacuum solution for the region $r>R$ is $T_{i j}=0$ for the standard GR case. Now it is important to note that as $r=$ $R$ is the boundary, and the exterior space-time may cease to be vacuum due to the presence of new emergent fields as a result of the $\theta$ sector. These extra fields result in anisotropy inside the self-gravitating system. So, the following final form will be taken from the above boundary condition (55) as,

$$
p(R)-\left(\theta_{1}^{1}\right)^{-}(R)=-\left(\theta_{1}^{1}\right)^{+}(R)
$$

where $p(R)=p^{-}(R)$. The condition coming in the Eq. (56) is known as the general expression for the second fundamental form linked with Einstein field equations as mentioned in Eq. (6). Now, the value of $\theta_{1}^{1}(R)$ is substituted into the condition (56) for the interior geometry from Eq. (34). So the second fundamental form can be written as follows,

$$
\begin{aligned}
& p(R)+\frac{\alpha}{8 \pi}\left[f(R)\left(\frac{v^{\prime}(R)}{R}+\frac{1}{R^{2}}\right)-\frac{\mu(R) h^{\prime}(R)}{R}\right] \\
& \quad=-\left(\theta_{1}^{1}\right)^{+}(R)
\end{aligned}
$$

where, $v^{\prime}(R) \equiv\left(\frac{\partial v^{-}}{\partial r}\right)_{r=R}$. The Eqs. (34), (53) and (54) are used in Eq. (57) to obtain $\left(\theta_{1}^{1}\right)^{+}(R)$ for the outer geometry, which yields,

$$
\begin{aligned}
& p(R)+\frac{\alpha f(R)}{8 \pi}\left(\frac{\nu^{\prime}(R)}{R}+\frac{1}{R^{2}}\right)-\frac{\alpha \mu(R) h^{\prime}(R)}{8 \pi R} \\
& =\frac{\alpha f^{*}(R)}{8 \pi}\left[\frac{2}{R^{2}}\left(\frac{M}{R}-\frac{Q^{2}}{R^{2}}\right) \frac{1}{\left(1-\frac{2 M}{R}+\frac{Q^{2}}{R^{2}}\right)}+\frac{1}{R^{2}}\right] \\
& \quad-\frac{\alpha\left(h^{*}(R)\right)^{\prime}}{8 \pi R}\left(1-\frac{2 M_{0}}{R}+\frac{Q^{2}}{R^{2}}\right)
\end{aligned}
$$

where, due to the extra source $\theta_{\mu v}$, the geometric deformation functions for exterior Reissner-Nordström solution (51) are $f^{*}(R)$ and $h^{*}(R)$ respectively. It is important to note that we must have $f^{*}(R)=0$ and $h^{*}(R)=0$ for satisfying the Reissner-Nordström solution (51) to be the exterior solution. Now, from Eq. (58) we can obtain,

$p(R)+\frac{\alpha f(R)}{8 \pi}\left(\frac{\nu^{\prime}(R)}{R}+\frac{1}{R^{2}}\right)-\frac{\mu(R) h^{\prime}(R)}{8 \pi R}=0$,

which can also be described as,

$p_{r}^{\text {tot }}(R)=p(R)-\theta_{1}^{1}(R)=0$. 


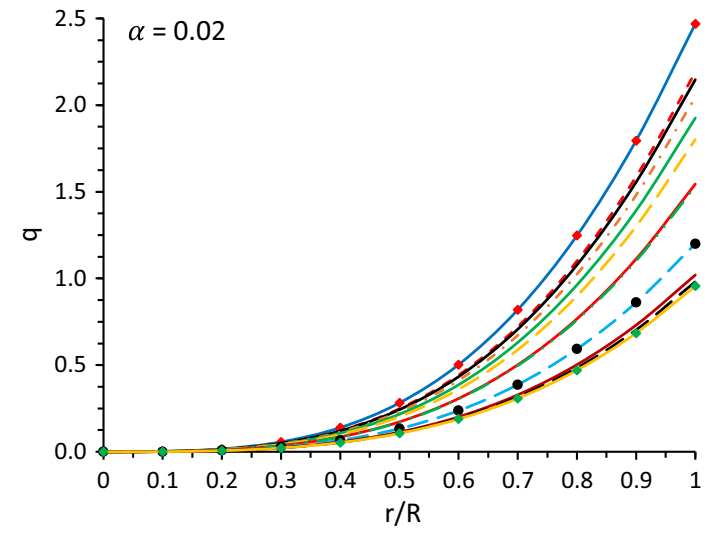

Fig. 2 The variation of electric charge $(q)$ versus radius $r / R$ by taking the numerical values of the constants $a=0.003, b=0.0000053$, $\beta=1.1$, and $\gamma=-0.001$. The description of the curves are given at the footnote

For determining the arbitrary constants involving the system, the conditions (53), (54) and (60) are necessary and sufficient. Now by using the Eqs. (26), (27), (38) and (53), (54), we obtain

$$
\begin{aligned}
& \frac{1}{1+a R^{2}+b R^{4}}+\alpha f(R)=\left[1-\frac{2 M}{R}+\frac{Q^{2}}{R^{2}}\right], \\
& B R^{2}+C+\alpha h(r)=\ln \left[1-\frac{2 M}{R}+\frac{Q^{2}}{R^{2}}\right] .
\end{aligned}
$$

Form Eqs. (61) and (62), we get

$$
\begin{aligned}
& C=\ln \left[\frac{1}{1+a R^{2}+b R^{4}}+\alpha f(R)\right]-B R^{2}-\alpha h(R), \\
& M=\frac{R}{2}\left[\frac{a R^{2}+b R^{4}}{1+a R^{2}+b R^{4}}-\alpha f(R)+\frac{Q^{2}}{R^{2}}\right]
\end{aligned}
$$

while the constant $B$ is calculated by using second fundamental form (60) that provides,

$$
B=\frac{B_{0}(R)+\sqrt{B_{1}(R)+8\left(R^{2}+a R^{4}+b R^{6}\right)\left[B_{2}(R)+a B_{3}(R)\right]}}{4 R^{2}\left(1+a R^{2}+b R^{4}\right)} .
$$

From Fig. 2, it is evident that for all the stars, the electric charge is zero at the center, and the charge increases sharply as one moves towards the surface while the magnitude of the charge is directly proportional to compactness $(u)$.

\section{The regular behavior of deformed gravitational potential together with deformation functions}

Since the regular behavior of deformed gravitational potential depends upon the deformation functions $f(r)$ and $h(r)$. As we look in Fig. 3 (left panel), the deformation function $f(r)$ is zero at the center and decreasing towards the boundary which yields $e^{-\lambda}=\mu+\alpha f(r)$ as a decreasing function of $r$ and then $e^{\lambda}$ will be increasing outward when $r$ increases as well as it is 1 at the center, see Fig. 4 (left panel). On the other hand, deformation function $h(r)$ monotonically decreasing and positive as well as non-zero at the center which can be clearly observed from Fig. 3 (right panel). Then in this situation, the deformed potential $v=\xi+\alpha h(r)$ (known as $g_{t t}$ component or temporal component of the space-time) may be decreasing outward, and obviously $e^{\nu(r)}$. But it is well-known that the modeling for any realistic selfgravitating stellar compact objects, the gravitational potential $v$ corresponding to spherically symmetric space-time must be monotonically increasing towards the boundary and positive finite at center of the stellar object. In the present scenario, as we see from Fig. 4 (right panel), the deformed gravitational $e^{v}$ corresponding to $g_{t t}$ component of the spherically space-time is monotonically increasing towards the surface and non-zero positive finite at the center which implies that the growth of $\xi$ is faster than $\alpha h(r)$. So, we concluded that the deformation function $h(r)$ for the temporal metric component can be a monotonically decreasing function of $r$ but the growth of $\alpha h(r)$ should be less than the growth of $g_{t t}$ component of seed space-time. Then, the present results strongly support that this approach is also more suitable to determine the deformation function $h(r)$ of temporal metric component for modeling of the compact stellar objects. Finally, we can say that both the deformed gravitational potentials $e^{\lambda}$ and $e^{\nu}$ together with deformation function $f(r)$ and $g(r)$ are regular and well-behaved throughout the model. ${ }^{4}$

\section{Physical properties of the charged anisotropic astrophysical model}

6.1 Physical behavior for $p_{r}^{\text {tot }}, p_{t}^{\text {tot }}, p_{r}^{\text {tot }}$, and $\Delta^{\text {tot }}$

The essential physical parameters such as $p_{r}^{\text {tot }}, p_{t}^{\text {tot }}, \rho^{\text {tot }}$, and $\Delta^{\text {tot }}$ are dependent on constant parameters $a, b, \beta, \gamma$ and they are fixed in such a way that all these could maintain the properties of a well behaved stellar model. As, it is seen from Fig. 5 that the total radial pressure $p_{r}^{\text {tot }}$ and total tangential pressure $p_{t}^{\text {tot }}$ both attain their highest value at the center and decrease monotonically as we shift towards the surface of the star models. Also, the total radial pressure decreases slightly steeper than the total tangential pressure. As observed in Fig. 6 (left

\footnotetext{
4 The description of the curves in Figs. 2, 3, 4, 5, 6, 7, 8, 9 and 10 are as follows: PSR J1416-2230 (solid blue with marker), Vela X-1 (dashedred), 4U 1608-52 (solid black), PSR J1903+327 (dashed-dot yellow), 4 U 1820-30 (solid green), Cen X-3 (long dashed yellow), EXO 1785248 (solid red), LMC X-4 (dashed-dot green), SMC X-1 (dashed blue with marker), SAX J1808.4-3658 (solid dark-red), 4U 1538-52 (long dashed black), Her X-1 (solid yellow with marker).
} 

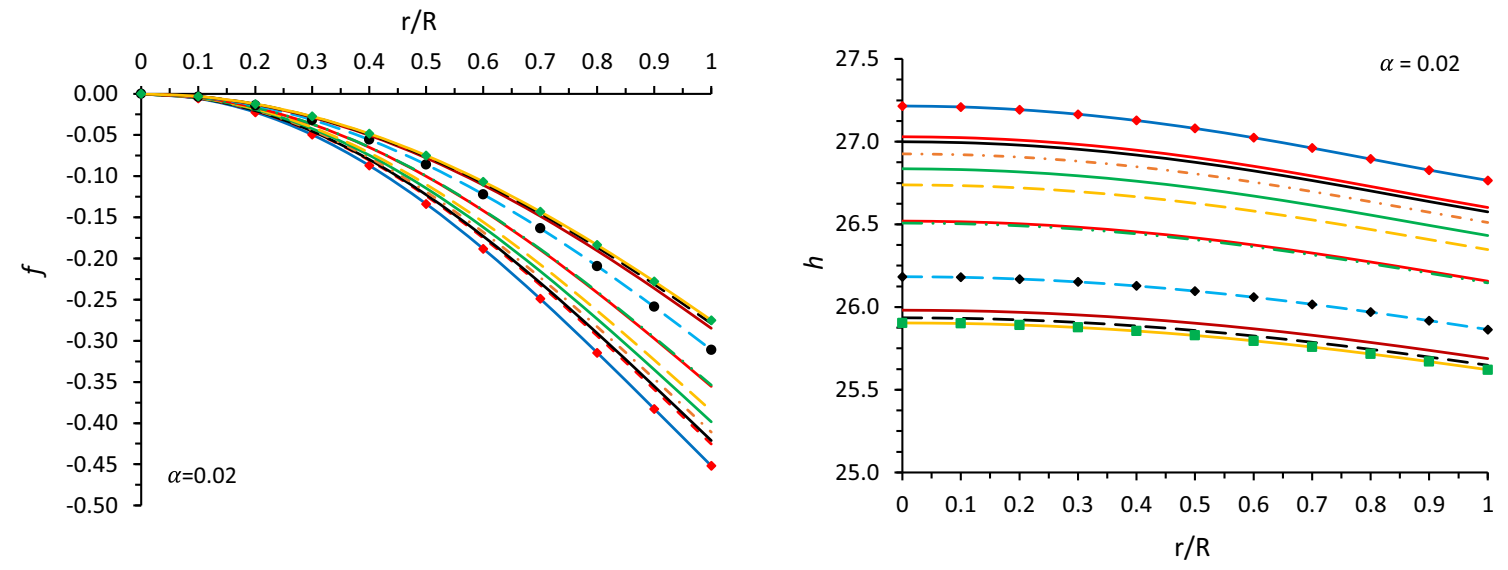

Fig. 3 The variations of deformation functions $f(r)$ and $h(r)$ versus radius $r / R$ by taking the numerical values of the constants $a=0.003$, $b=0.0000053, \beta=1.1$, and $\gamma=-0.001$. The description of the curves are given at the footnote

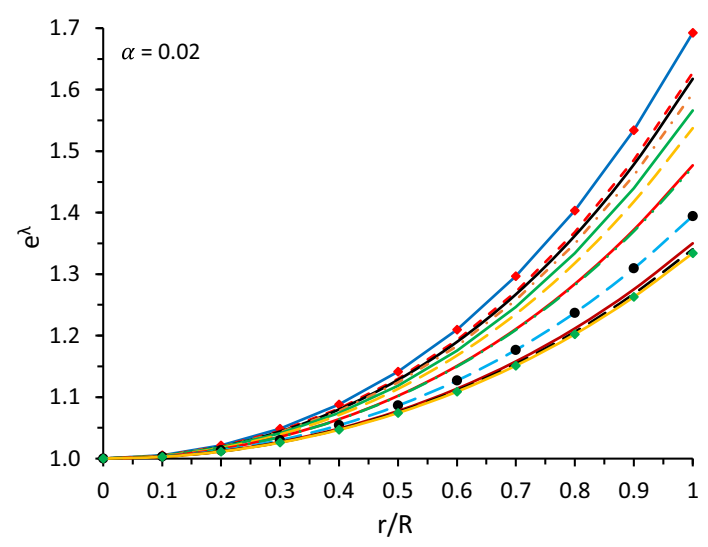

Fig. 4 The behavior of gravitational potentials $e^{\lambda(r)}$ (left panel) and $e^{\nu(r)}$ (right panel) versus radial coordinate $r / R$ by taking the numerical values of the constants $a=0.003, b=0.0000053, \beta=1.1$, and $\gamma=-0.001$

panel), the total energy density $\rho^{\text {tot }}$ has its maximum value at the center and decreases monotonically as we move to the surface boundary, which suggests that the energy density is maximum in the core of the compact stellar object. Also, it is observed that both the pressure components are directly proportional with compactness or $M / R$ ratio, whereas, the energy density is inversely proportional with the compactness as can be seen from Table 1 and Figs. 5 and 6. Now while studying the anisotropic factor $\Delta^{\text {tot }}$ from Fig. 6 (right panel), we find that the anisotropic factor is zero at the center, i.e. the radial and tangential pressure is same at the center, and as we move towards the surface, the $p_{r}^{\text {tot }}$ and $p_{t}^{\text {tot }}$ tend to bifurcate more from each other and then anisotropy increases and attains the maximum near the surface. This monotonically increasing, finite and continuous anisotropic factor implies that the anisotropic force $\left(F_{a}=2 \Delta / r\right)$ is directed outwards which plays an important role to prevent gravitational collapse of the self-gravitating compact objects.

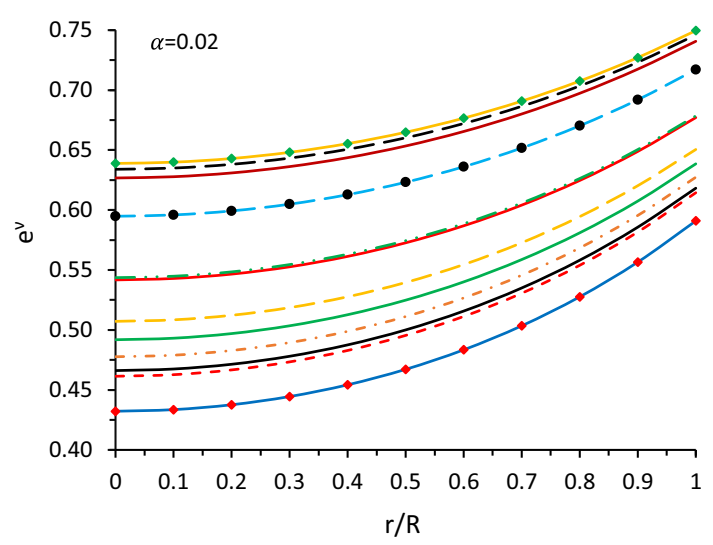
6.2 Energy conditions

Here, the energy conditions are discussed by considering of the relativistic and classical gravitational field theories. These energy conditions are local inequalities obeying some specific restrictions and possessing certain relation between pressure and matter density. Although, many significant physical constraints have been suggested, including the singularity theorems [79], positive mass theorem [80], the censorship theorem [81,82], also several constraints on black hole surface gravity [83], but the most significant ones are the energy conditions and among them, our focus will be only on the below mentioned five conditions, namely (i) the null energy condition (NEC), (ii) the weak energy condition (WEC), and (iii) the strong energy condition (SEC), (iv) dominant energy condition, and (v) Trace energy condition.

$$
\begin{aligned}
& \text { NEC }: \quad \rho^{\text {tot }}+\frac{q^{2}}{8 \pi r^{4}} \geq 0, \\
& \text { WEC }: \quad \rho^{\text {tot }}+p_{r}^{\text {tot }} \geq 0, \quad \rho^{\text {tot }}+p_{t}^{\text {tot }}+\frac{q^{2}}{4 \pi r^{4}} \geq 0,
\end{aligned}
$$




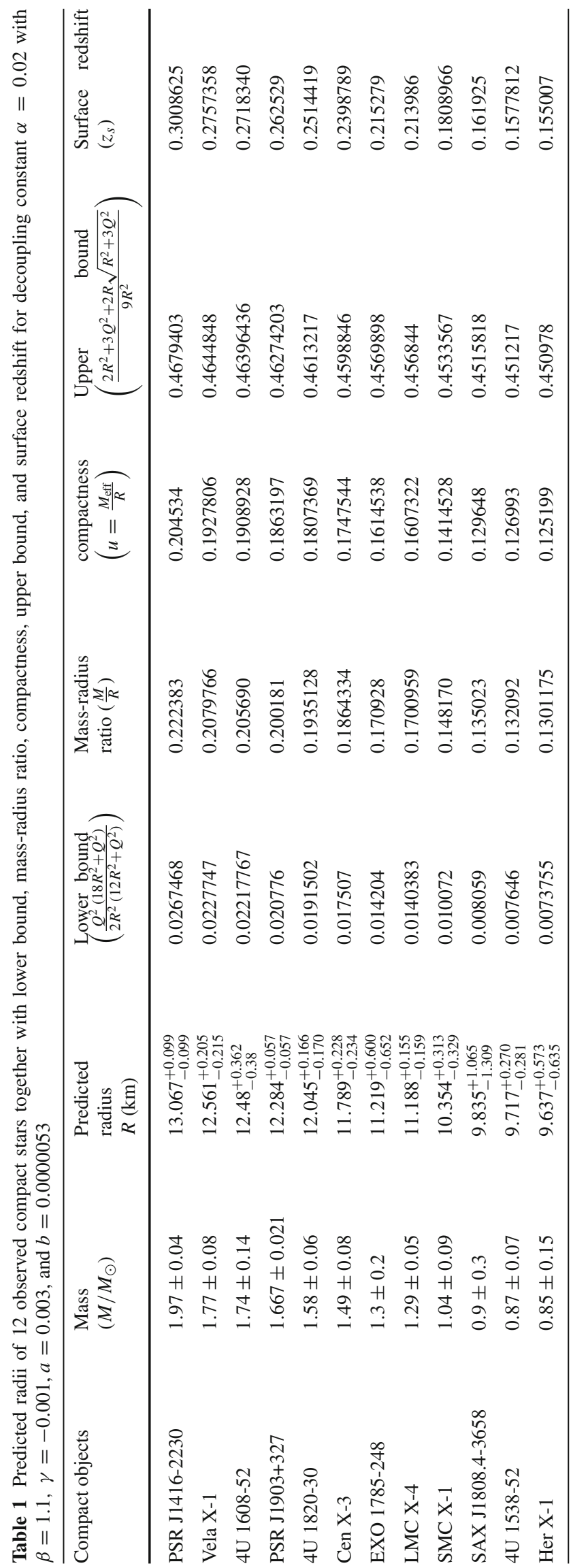



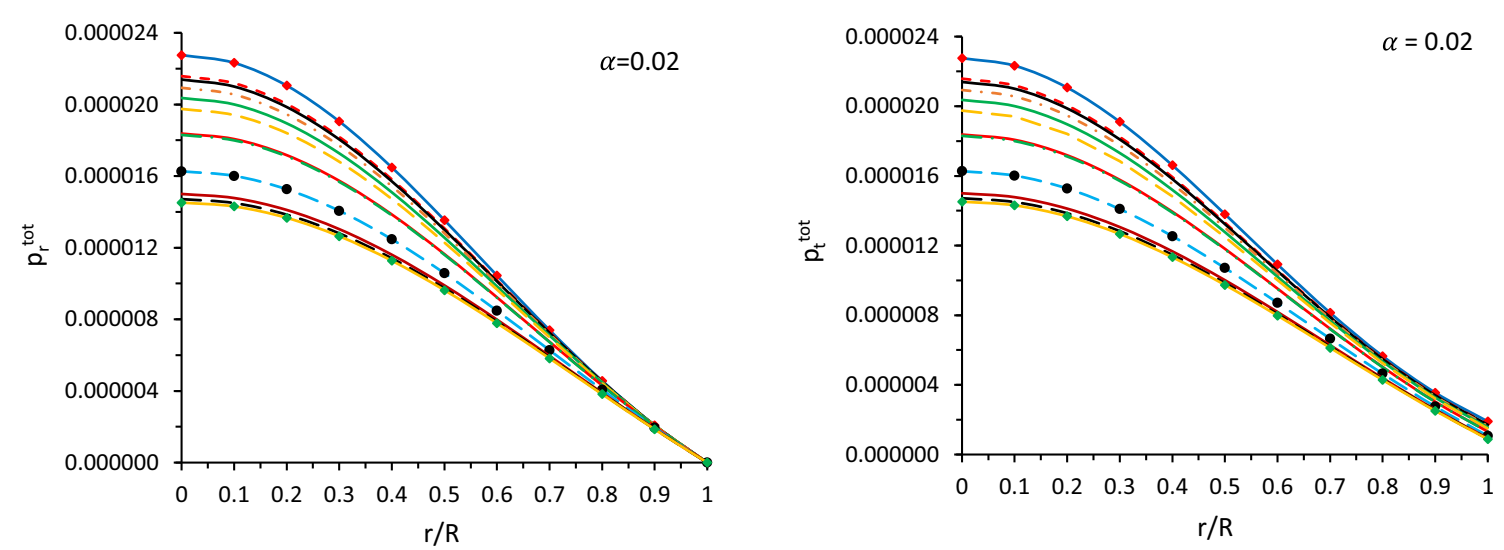

Fig. 5 The behavior of total radial pressure ( $\left.p_{r}^{\text {tot }}\right)$ and total tangential pressure $\left(p_{t}^{\text {tot }}\right)$ versus radial coordinate $r / R$ by taking the numerical values of the constants $a=0.003, b=0.0000053, \beta=1.1$, and $\gamma=-0.001$
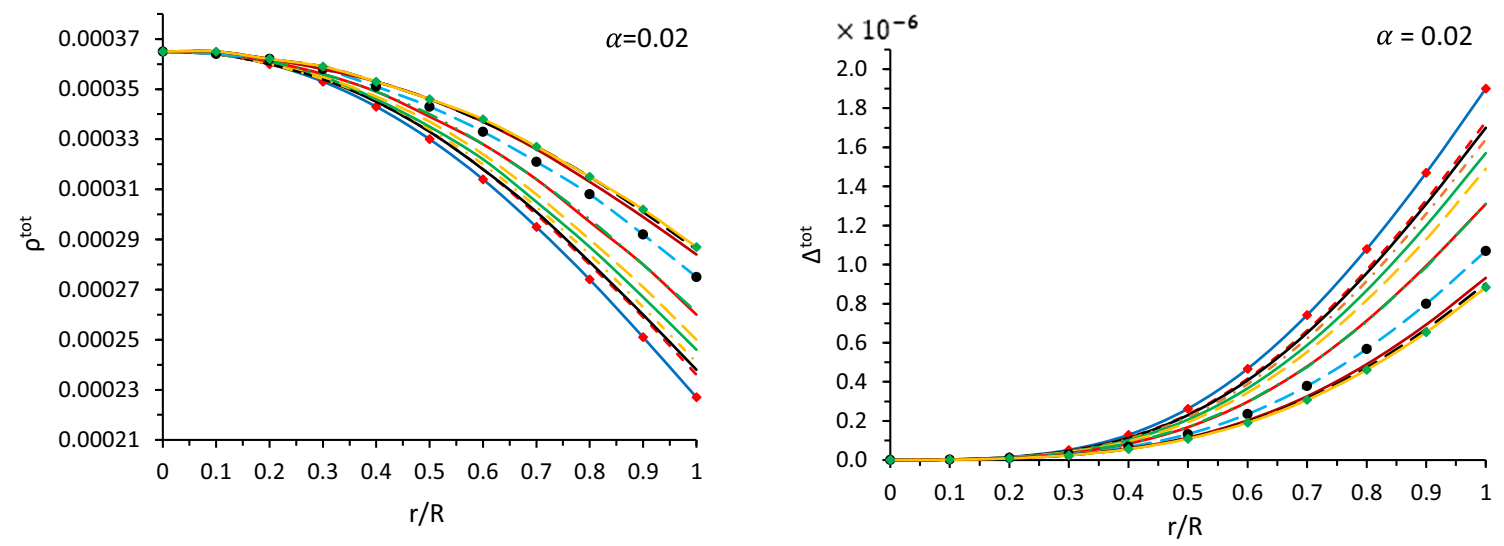

Fig. 6 The behavior of total energy density $\left(\rho^{\text {tot }}\right)$ and total anisotropy $\left(\Delta^{\text {tot }}\right)$ versus radial coordinate $r / R$ by taking the numerical values of the constants $a=0.003, b=0.0000053, \beta=1.1$, and $\gamma=-0.001$
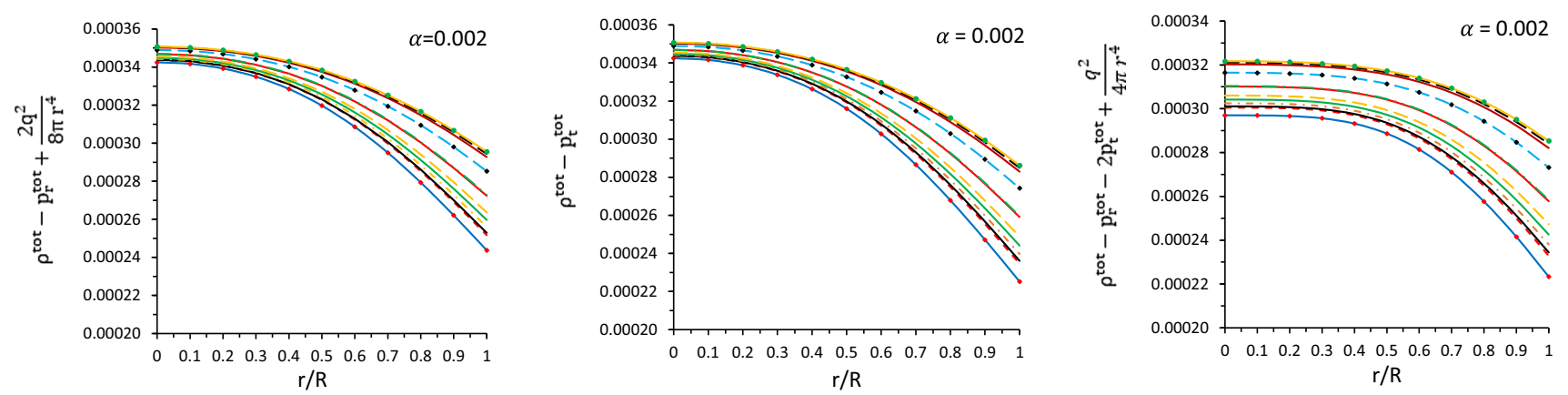

Fig. 7 The behavior of dominant energy conditions and trace energy condition versus radial coordinate $r / R$ by taking the numerical values of the constants $a=0.003, b=0.0000053, \beta=1.1$, and $\gamma=-0.001$

$\mathrm{SEC}: \rho^{\text {tot }}+p_{r}^{\text {tot }}+2 p_{t}^{\text {eff }}+\frac{q^{2}}{4 \pi r^{4}} \geq 0$.

$\mathrm{DEC}: \quad \rho^{\mathrm{tot}}-p_{r}^{\mathrm{tot}}+\frac{q^{2}}{4 \pi r^{4}} \geq 0, \quad \rho^{\mathrm{tot}}-p_{t}^{\mathrm{tot}} \geq 0$,

TEC : $\quad \rho^{\text {tot }}-p_{r}^{\text {tot }}-2 p_{t}^{\text {tot }} \geq 0$.

We would like to mention here that $\rho^{\text {tot }}, p_{r}^{\text {tot }}, p_{t}^{\text {tot }}$ and $q^{2}$ are positive throughout at each point within the stellar objects (see Figs. 2, 3, 4, 5 and 6) then obviously the inequalities (66), (67) and (68) will automatically satisfy everywhere within the stellar objects. So, it is enough to discuss the analysis of the last two inequalities (69) and (70) through the graphical representation. In Fig. 7, we show the trends of the DEC and TEC, and observed that both energy conditions are positive throughout at each point of the stellar interiors. Therefore, all the energy conditions are satisfying within the models. 


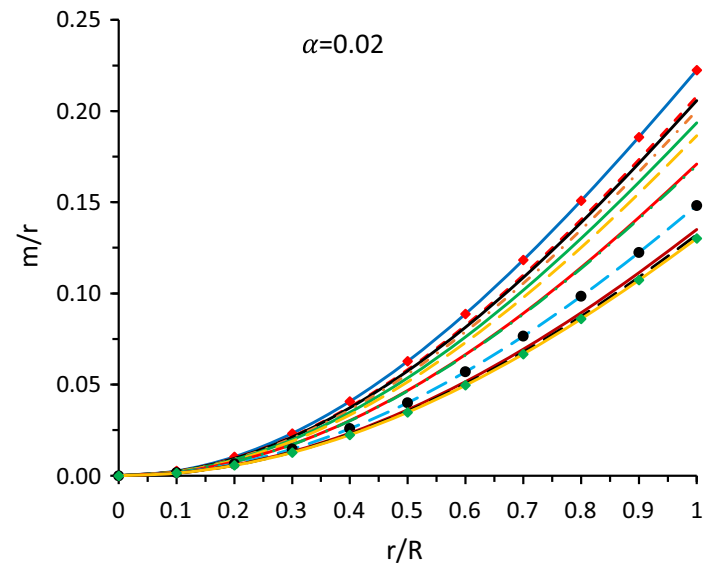

Fig. 8 The behavior of mass-radius ratio $(m / r)$ versus radial coordinate $r / R$ by taking the numerical values of the constants $a=0.003$, $b=0.0000053, \beta=1.1$, and $\gamma=-0.001$

\subsection{Compactness (mass-radius) relation and surface gravitational redshift}

The compactness (mass-radius ratio) is a very important ingredient to discuss for any stable self-gravitating compact star model. On the other hand, the effective mass-radius ration also plays an important role to decide the upper limit surface red-shift of the compact objects. It is noted that the effective mass of the compact object is the same as total mass in the uncharged matter distributions while it is different in charged matter distribution scenario, which can be understand by following definitions:

- The effective mass in standard GR for perfect fluid/anisotropic uncharged matter distribution can be determinedbreak by the formula,

$$
\left[\hat{M}_{0}\right]_{\mathrm{eff}}=4 \pi \int_{0}^{R} r^{2} \rho(r) d r=\frac{R}{2}[1-\mu(R)]=\hat{M}_{0} .
$$

- However, the effective mass in standard GR for perfect fluid/anisotropic charged matter distribution can be obtained by,

$$
\left[M_{0}\right]_{\mathrm{eff}}=4 \pi \int_{0}^{R}\left(\rho+\frac{q^{2}}{8 \pi r^{4}}\right) r^{2} d r=\frac{R}{2}[1-\mu(R)],
$$

where $\hat{M}_{0}$ and $M_{0}$ are the total mass of the compact object of radius $R$ for uncharged and charged matter distribution, respectively in the absence of gravitational decoupling. ${ }^{5}$ Then effective mass under gravitational decoupling can be given as,

$$
M_{\mathrm{eff}}=\left[M_{0}\right]_{\mathrm{eff}}-\frac{\alpha R}{2} f(R) .
$$

In this connection, the maximum allowable the mass-radius ratio was proposed by Buchdahl [84] in the framework of isotropic perfect fluid distribution taking into account of decreasing density as $\frac{\hat{M}_{0}}{R} \leq \frac{4}{9}$. But if the electric field is introduced inside the matter distribution, then obviously this maximum allowable mass-radius ratio will also change. In this concern, Andreasson [85] and Bohmer-Harko [86] proposed the upper and lower bounds of mass-radius ratio in the presence of an electric charge, respectively. In view of the above, these upper bounds in presence of gravitational decoupling can be written as:

$$
\begin{aligned}
& \frac{M}{R} \leq \frac{2}{9}+\frac{3 Q^{2}+2 R \sqrt{R^{2}+3 Q^{2}}}{9 R^{2}} \quad \text { (Upper Bound), } \\
& \frac{M}{R} \geq \frac{Q^{2}\left(18 R^{2}+Q^{2}\right)}{2 R^{2}\left(12 R^{2}+Q^{2}\right)} \quad \text { (Lower Bound). }
\end{aligned}
$$

We would like to highlight that the gravitational decoupling will also introduce some effects on the total mass. Since $f(r)$ is non-positive throughout the star then we always get $M \geq$ $M_{0}$ for fixed radius $R$. Moreover, it is obvious that the limit of the upper and lower bound will also change in the presence of the gravitational decoupling. ${ }^{6}$ Moreover, the surface redshift $z_{s}$ of the charged compact stars can be calculated by the formula,

$$
\begin{aligned}
& \mathrm{z}_{s}=(1-2 u)^{-1 / 2}-1 \\
& \text { where, } u \equiv \frac{M_{\mathrm{eff}}}{R}=\frac{\left[M_{0}\right]_{\mathrm{eff}}}{R}-\frac{\alpha R}{2} f(R) .
\end{aligned}
$$

This shows that the decoupling parameter $\alpha$ will also introduce some effects on the surface red-shift value. The variations of the mass-radius ratio $(m / r)$ for 12 compact objects have been shown in Fig. 8. The $m / r$ ratio is increasing and attains its maximum value at the boundary of stellar objects. On the other hand, In Table 1, we presented the data values of the lower and upper bound for $M / R$ as mentioned in Eqs. (74) and (75) together with $M_{\mathrm{eff}} / R$ and $z_{s}$ of each compact objects for $\alpha=0.02$. It can be clearly observed that $M / R$ satisfies both inequalities (74) and (75). Then obviously $M_{\text {eff }} / R$ will also lie inside the lower and upper bound

\footnotetext{
5 However, In the presence of gravitational decoupling, the total mass $(\hat{M})$ and $(M)$ corresponding to uncharged and charged matter distribution respectively, can be written as: $\hat{M}=\hat{M}_{0}-\frac{\alpha R}{2} f(R)=$ $4 \pi \int_{0}^{R} r^{2} \rho^{\mathrm{tot}}(r) d r=\frac{R}{2}\left[1-e^{-\lambda(R)}\right]$ and $M=M_{0}-\frac{\alpha R}{2} f(R)=$ $4 \pi \int_{0}^{R} r^{2}\left[\rho^{\text {tot }}(r)+\frac{q^{2}}{8 \pi r^{2}}\right] d r=\frac{R}{2}\left[1-e^{-\lambda(R)}+\frac{Q^{2}}{R^{2}}\right]$.

${ }^{6}$ This change will happen due to change in total charge-radius ratio.
} 

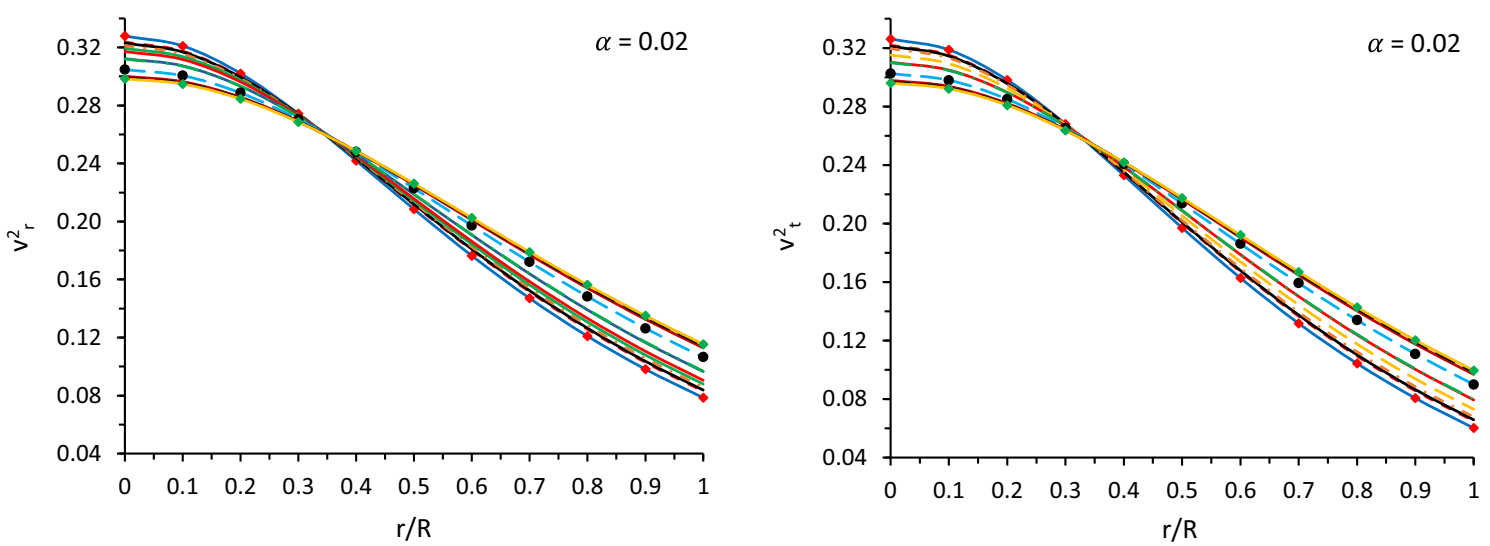

Fig. 9 The behavior of radial velocity $\left(v_{r}^{2}\right)$ and tangential velocity $\left(v_{t}^{2}\right)$ versus radial coordinate $r / R$ by taking the numerical values of the constants $a=0.003, b=0.0000053, \beta=1.1$, and $\gamma=-0.001$

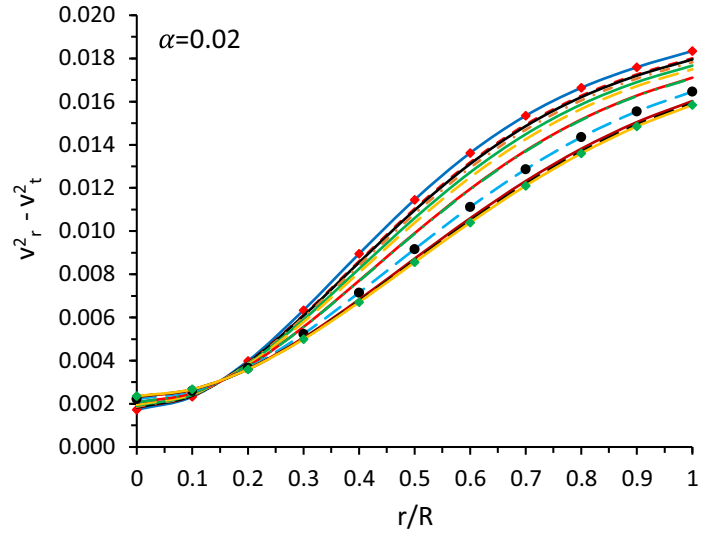

Fig. 10 The behavior of stability factor $\left(v_{r}^{2}-v_{t}^{2}\right)$ versus radial coordinate $r / R$ by taking the numerical values of the constants $a=0.003$, $b=0.0000053, \beta=1.1$, and $\gamma=-0.001$

since $M>M_{\text {eff. Also, the calculated surface red-shift values }}$ of the objects for different values of decoupling parameter $\alpha$ as mentioned in Tables 1 and 3 are compatible with the range proposed by Ivanov [14] and Bowers-Liang [87].

\subsection{Stability and dynamical equilibrium}

\subsubsection{Velocity of sound and stability via. cracking concept}

In this section, we discussed the status of the velocity of sound within the stellar models. For any realistic stellar object, the velocity of sound must be lesser than the speed of light i.e. $v_{r}^{2}$ and $v_{t}^{2}$ must lie in $[0,1]$ which is known as the causality condition. We observe from Fig. 9, the radial velocity $\left(v_{r}^{2}\right)$ and tangential velocity $\left(v_{t}^{2}\right)$ are less than unity, which shows a realistic model.

Now we investigate the stability of the solution using Herrera [88] and Abreu's [89] criterion. These criterion mainly depend upon the subliminal radial and tangential velocity of sounds which can provide whether the system is stable or unstable under cracking instability. The cracking method is a tool to study instability of the self-gravitating solution in the presence of anisotropic matter distributions. However, this tool can be easily described through the subliminal speed of pressure waves. According to Abreu, the cracking concept can be understand by the magnitude of perturbations in anisotropy $\left(\left|\delta \Delta^{\text {tot }}\right|\right)$ and density $\left(\delta \rho^{\text {tot }}\right)$ ratio i.e. $\left|\frac{\delta \Delta^{\text {tot }}}{\delta \rho^{\text {tot }}}\right|$ as,

$$
\left|\frac{\delta \Delta^{\mathrm{tot}}}{\delta \rho^{\mathrm{tot}}}\right| \simeq\left|\frac{\delta\left(p_{t}^{\mathrm{tot}}-p_{r}^{\mathrm{tot}}\right)}{\delta \rho^{\mathrm{tot}}}\right| \simeq\left|\frac{\delta p_{t}^{\mathrm{tot}}}{\delta \rho^{\mathrm{tot}}}-\frac{\delta p_{r}^{\mathrm{tot}}}{\delta \rho^{\mathrm{tot}}}\right| \simeq\left|v_{t}^{2}-v_{r}^{2}\right|
$$

Since the stellar models are satisfying the causality condition, then we have $0 \leq v_{r}^{2} \leq 1$ and $0 \leq v_{t}^{2} \leq 1$. But for any physically realistic stable models, the magnitude of perturbations in anisotropy should always be less than those in density i.e. $\left|\delta \Delta^{\text {tot }}\right| \leq\left|\delta \rho^{\text {tot }}\right| \Longrightarrow\left|v_{t}^{2}-v_{r}^{2}\right| \leq 1$, however we get an unstable region when $\left|\frac{\delta \Delta^{\mathrm{tot}}}{\delta \rho^{\mathrm{tot}}}\right|>0$. Then we can distinguish the stable and unstable regions via. following inequality,

$0 \leq\left|v_{t}^{2}-v_{r}^{2}\right| \leq 1=\left\{\begin{array}{l}-1 \leq v_{t}^{2}-v_{r}^{2} \leq 0 \\ 0<v_{t}^{2}-v_{r}^{2} \leq 1\end{array}\right.$

In view of above, the inequality $-1 \leq v_{t}^{2}-v_{r}^{2} \leq 0$ provides a stable region while $0<v_{t}^{2}-v_{r}^{2} \leq 1$ gives an unstable region. On the other way, we can say that those regions, where no change in the sign for the inequality $-1 \leq v_{t}^{2}-v_{r}^{2} \leq 0$, is called the stable regions. Therefore, we show the graphical analysis for the present self-gravitating solution to find the potentially stable region within the star model. From Fig. 10 it is observed that the system presents all the regions completely stable. 


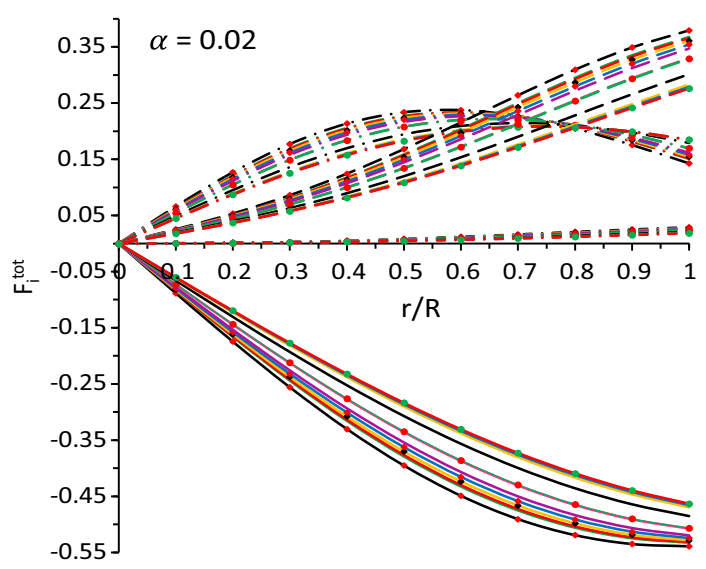

Fig. 11 The behavior of different forces $F_{g}^{\text {tot }}$ (solid lines), $F_{h}^{\text {tot }}$ (long dash-dotted lines), $F_{a}^{\text {tot }}$ (small dash-dotted lines) and $F_{e}^{\text {tot }}$ (long dashed lines) versus radial coordinate $r / R$ by taking the numerical values of the constants $a=0.003, b=0.0000053, \beta=1.1$, and $\gamma=-0.001$

\subsubsection{Hydrostatic equilibrium through generalized \\ Tolman-Oppenheimer-Volkoff (TOV) equation under extended gravitational decoupling}

For any realistic stellar structure, It is necessary to satisfy the Tolman-Oppenheimer-Volkoff (TOV) equation for the hydrostatic equilibrium. The generalized TOV equation (conservation equation) in the framework of gravitational decoupling can be obtained by a covariant derivative of total energy momentum tensor i.e. $\nabla^{j} T_{i j}^{\text {tot }}=0$ gives,

$$
\begin{aligned}
& p^{\prime}+\frac{\xi^{\prime}}{2}(\rho+p)-\frac{q q^{\prime}}{4 \pi r^{4}}+\alpha\left[\frac{v^{\prime}}{2}\left(\theta_{0}^{0}-\theta_{1}^{1}\right)+\frac{2}{r}\left(\theta_{2}^{2}-\theta_{1}^{1}\right)\right. \\
& \left.-\left(\theta_{1}^{1}\right)^{\prime}+\frac{h^{\prime}}{2}(p+\rho)\right]=0 .
\end{aligned}
$$

It is noted that if $\alpha=0$, then the above conservation equation leads the standard TOV equation in GR for charged matter distribution. To satisfy this conservation equation (79), we spilt this TOV equation into different forces like $F_{h}^{\text {tot }}, F_{g}^{\text {tot }}$, $F_{a}^{\text {tot }}$, and $F_{e}^{\text {tot }}$ such that $F_{h}^{\text {tot }}+F_{g}^{\text {tot }}+F_{a}^{\text {tot }}+F_{e}^{\text {tot }}=0$, where

$$
\begin{aligned}
F_{h}^{\mathrm{tot}} & =-p^{\prime}+\alpha\left(\theta_{1}^{1}\right)^{\prime}, \\
F_{g}^{\mathrm{tot}} & =-\frac{\xi^{\prime}}{2}(\rho+p)-\frac{\alpha v^{\prime}}{2}\left(\theta_{0}^{0}-\theta_{1}^{1}\right)-\frac{\alpha h^{\prime}}{2}(p+\rho)(81) \\
F_{a}^{\mathrm{tot}} & =-\frac{2 \alpha}{r}\left(\theta_{2}^{2}-\theta_{1}^{1}\right), \\
F_{e}^{\mathrm{tot}} & =\frac{q q^{\prime}}{4 \pi r^{4}} .
\end{aligned}
$$

We have plotted the Fig. 11 for different forces as mentioned above corresponding to 12 stars (see the footnote for details). From this figure, we observe that the gravitational force is strongly acting inward direction while other forces like anisotropic force, hydrostatic force, and electric force are acting in outward directions. The hydrostatic force is increasing and attains its maximum value at the inside the point of

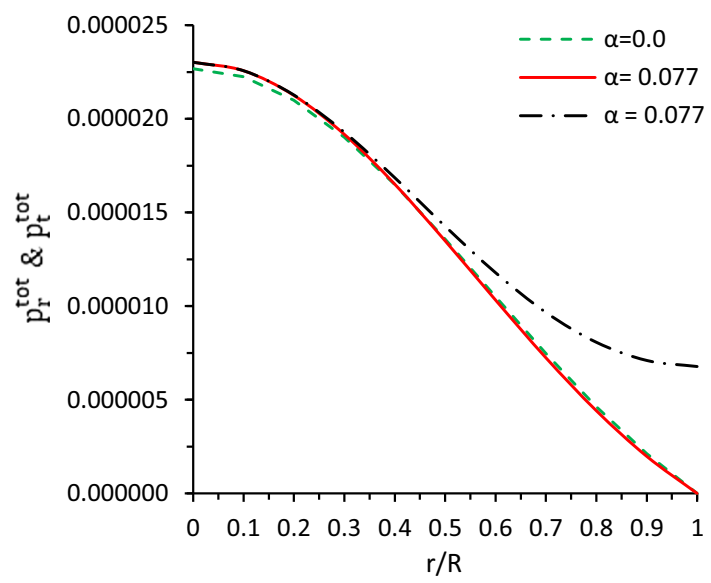

Fig. 12 The behavior of total radial and tangential pressures ( $p_{r}^{\text {tot }}$ and $p_{t}^{\text {tot }}$ ) versus radial coordinate $r / R$ by taking the numerical values of the constants $a=0.003, b=0.0000053, \beta=1.1$, and $\gamma=-0.001$. The description of the figure as follows: (i) the small dash green curve for $p(r)=p_{r}^{\text {tot }}(r)=p_{t}^{\text {tot }}(r)$ corresponding to $\alpha=0.0$, since $\alpha=0.0$ leads an isotropic charged matter distribution, (ii) solid red curve for $p_{r}^{\text {tot }}(r)$ corresponding to $\alpha=0.077$, and (iii) black long dashed-dot curve for $p_{t}^{\text {tot }}(r)$ for $\alpha=0.077$

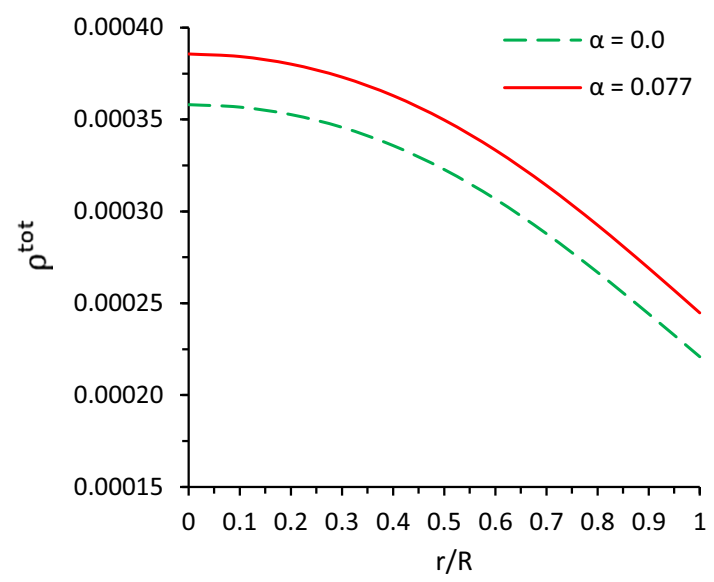

Fig. 13 The variation of total energy density $\left(\rho^{\text {tot }}\right)$ versus radial coordinate $r / R$ for different values of decoupling constant. The same constant values have been taken to plot this graph as used in Fig. 12

the stellar objects when $r / R \simeq 0.6$ and starts decreasing after this point, which implies that hydrostatic force $\left(F_{h}\right)$ prevents the gravitational force maximum at this point to avoid the gravitational collapse of the compact star to a point singularity, and the point $r / R \simeq 0.6$ represents the critical value for the present choice of the values for the parameters involved in the solution. However, $F_{g}^{\text {tot }}, F_{a}^{\text {tot }}$ and $F_{e}^{\text {tot }}$ are increasing monotonically throughout the stellar objects. Furthermore, we also noticed that electric force plays a major role to balance the system near the surface against the gravitational force, and the anisotropic force has very less impact to achieve the hydrostatic equilibrium. Finally, the overall conclusion is that the gravitational force is balanced by joint 
action of anisotropic force, hydrostatic force, and electric force such that $F_{g}^{\text {tot }}+F_{a}^{\text {tot }}+F_{e}^{\text {tot }}+F_{g}^{\text {tot }}=0$. In this way, we achieved the hydrostatic equilibrium of the solution for each obtained compact objects. ${ }^{7}$

\section{Impact of coupling constant $\alpha$ on the thermodynamical observables}

Now observing the impact of the coupling constant $\alpha$ on thermodynamical observables, we plotted the Figs. 12, 13 and 14 and prepare Tables 2 and 3 for particular star PSR J14162230. To show this, we find that when $\alpha=0$, the radial, and tangential pressure components are the same and the matter distribution is isotropic. But when the $\alpha$ starts to increase, the pressure components start to bifurcate and in Fig. 12, as we can see, for $\alpha=0.77$, the radial pressure (denoted by the red solid line) and tangential pressure (denoted by the dot-dashed black line) deviates a lot and this deviation becomes maximum near the surface. So we can say, the decoupling parameter $\alpha$ introduces anisotropy inside the matter distribution and when the value of $\alpha$ increases, the anisotropy becomes more and it grows as one moves from the center and is most noticeable near the surface. Also, as $\alpha$ increases, the energy density increases, which is directly proportional with $\alpha$ and the variation is slightly higher near the center and decreases very slightly near the boundary. Also, Fig. 14 (left panel) shows that the electric charge $q$ varies inversely with $\alpha$, and the variation increases as we move towards the surface. The variation of mass-radius ratio $(\mathrm{m} / \mathrm{r})$ within the stellar interior has been shown in Fig. 14 (right panel). From this figure, It is clear that the mass-radius ratio varies proportionally with the $\alpha$, and the variation is most noticeable near the surface, in this case also. On the other hand, from Table 2, we notice that the central pressure $\left(p_{c}^{\text {tot }}\right)$, central and surface density $\left(\rho_{c}^{\text {tot }}\right.$ and $\left.\rho_{s}^{\text {tot }}\right)$ are increasing when $\alpha$ increases but the total charge on the boundary $Q$ decreases when $\alpha$ increases. Moreover, The Table 3 shows that lower bound, mass-radius ratio $(M / R)$, upper bound, compactness $\left(u=M_{\mathrm{eff}} / R\right)$ and surface redshift for the same particular star PSR J1416-2230. As we can see that the $M / R$ and $M_{\text {eff }} / R$ are lying between the lower and upper bound for each of the obtained values of $\alpha$. Also both $M / R$ and $\hat{M}_{\text {eff }} / R$ are increasing when $\alpha$ increases then obviously the surface red-shift $\left(z_{s}\right)$ will increase with $\alpha$ also.

we would like to highlight one interesting point here that the gravitational decoupling, especially geometric deformation along with radial component of the metric function

\footnotetext{
7 The description of curves in the Fig. 11 are as follows: PSR J14162230 (black with marker), Vela X-1 (green), 4U 1608-52 (red), PSR J1903+327 (yellow with marker), 4U 1820-30 (blue with marker), Cen X-3 (purple), EXO 1785-248 (magenta), LMC X-4 (green with marker), SMC X-1 (black), SAX J1808.4-3658 (yellow), 4U 1538-52 (blue), Her $\mathrm{X}-1$ (red with marker).
}

i.e. along with $g_{00}$ component, modifies the total mass of the objects. The total mass of the gravitationally decoupled object is $M=M_{0}-\frac{\alpha R}{2} f(R)$, where $M_{0}$ is a total mass of the charged object for seed space-time. As clearly indicated in Fig. 3 (left panel) that deformation function $f$ is negative for all $r>0$, which implies that the factor $-\frac{\alpha R}{2} f(R)$ will be positive, and then this factor will enhance the total mass of the objects. As we know that any massive object warps and curving the space-time. Since the gravitational field is basically a curving of the space-time, which means that more massive objects create a stronger gravitational field. On the other hand, the gravitational time dilation occurs because the objects with lot of mass create a strong gravitational field (i.e. stronger gravity), then it is clear that the gravitationally decoupled models may produce a stronger gravitational field, i.e. the stronger the gravity, the more curving space-time, and the slower time itself proceeds. On the other hand, the CGD introduces the transformation along with the temporal components i.e. along $g_{t t}$ of the space-time, which affects the proper time around astrophysical objects. From the concept of general relativity, the slowed time in higher gravitational potential is given by

$\Delta t=\sqrt{g_{t t}} d t=\sqrt{e^{v(r)}} d t$.

Here $d t$ is the time measured at infinity and $\Delta t$ near the object. And in the weak field approximation we know that $g_{t t}=1+2|\Phi|$. Therefore, at far away distances $\Phi \rightarrow 0$ and hence no gravitational time slowed. However, as the gravitational potential increases near the object the slowed time $\Delta t$ increases. Hence, the dilated time can be written as

$d t=\frac{\Delta t}{\sqrt{g_{t t}}}=\frac{\Delta t}{\sqrt{e^{\nu(r)}}}$

In Fig. 15, one can see that the dilated time reduces to far away distances and increases near the surface, and continue till the center of the object. This is because assuming a spherically symmetric uniform density solid object the potential at the center is $3 / 2$ times higher than that of the surface. Extending this analogy to compact objects, the density increases inward towards the center and hence the potential. This leads to an increase in time dilation at the center compared to the surface.

\section{Discussion and conclusion}

In this paper, the extended gravitational decoupling by means of complete geometric deformation (CGD) approach has been utilized to find an anisotropic spherical solution in the presence of electric charge. To do this, we have modified Einstein-Hilbert action by introducing an extra action $S_{\theta}$ corresponding the extra source. The field equations for the 

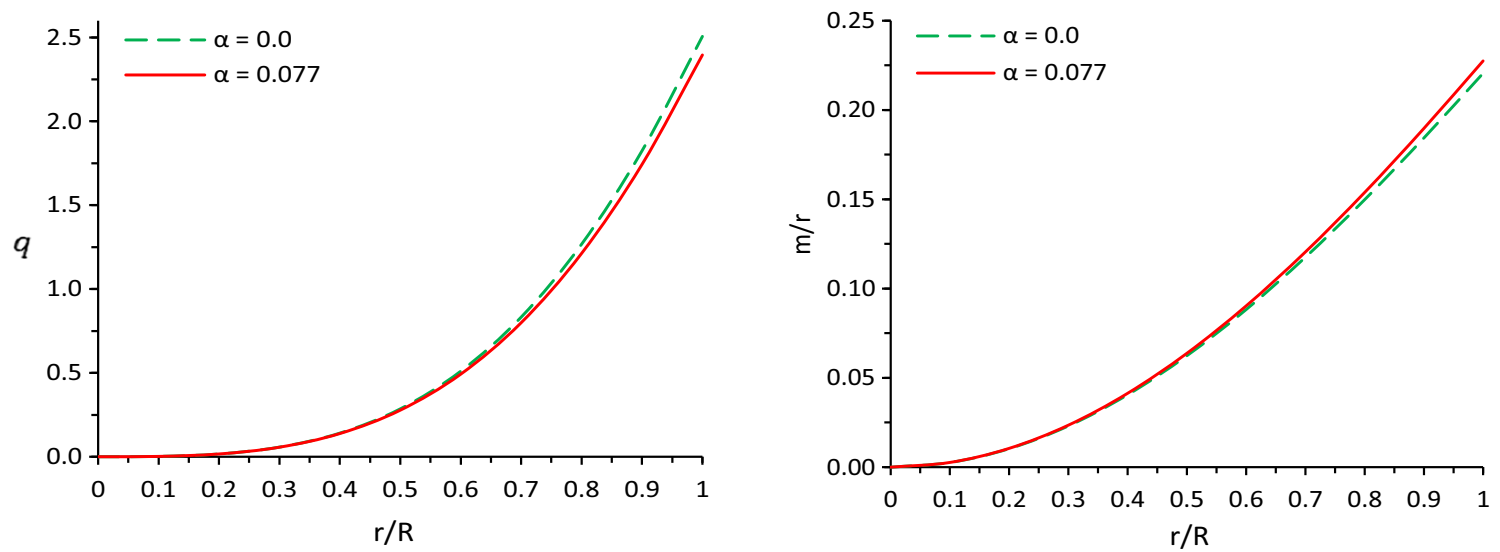

Fig. 14 The variation of electric charge $(q)$ (left panel) and mass-radius ratio $(m / r)$ (right panel) versus radial coordinate $r / R$ for different values of $\alpha$. For plotting this graph, we have used same numerical values of the constants as used in Fig. 12

Table 2 Numerical values of physical parameters central density $\left(\rho_{c}^{\text {tot }}\right)$, surface density $\left(\rho_{s}^{\text {tot }}\right)$, central pressure $\left(p_{c}^{\text {tot }}\right)$, constant $B$ and $C$ for star PSR J1416-2230 with different values of $\alpha$ with $\beta=1.1, \gamma=-0.001, a=0.003$, and $b=0.0000053$

\begin{tabular}{|c|c|c|c|c|c|c|c|}
\hline Values $(\alpha)$ & Predicted radius $(R)(\mathrm{km})$ & $\rho_{c}^{\mathrm{tot}}\left(\mathrm{gm} / \mathrm{cm}^{3}\right)$ & $\rho_{s}^{\text {tot }}\left(\mathrm{gm} / \mathrm{cm}^{3}\right)$ & $p_{c}^{\text {tot }}\left(\right.$ dyne $\left./ \mathrm{cm}^{2}\right)$ & Electric charge Q (in coulomb) & $B\left(\mathrm{~km}^{-2}\right)$ & $C$ \\
\hline 0.0 & $13.175_{-0.1}^{+0.1}$ & $4.83300 \times 10^{14}$ & $2.98154 \times 10^{14}$ & $2.75419 \times 10^{34}$ & $2.9216 \times 10^{20}$ & 0.00178 & -0.82887 \\
\hline 0.02 & $13.067_{-0.099}^{+0.099}$ & $4.92966 \times 10^{14}$ & $3.06544 \times 10^{14}$ & $2.76491 \times 10^{34}$ & $2.8784 \times 10^{20}$ & 0.00188 & -1.38309 \\
\hline 0.04 & $12.962_{-0.098}^{+0.097}$ & $5.02632 \times 10^{14}$ & $3.14911 \times 10^{14}$ & $2.77580 \times 10^{34}$ & $2.8428 \times 10^{20}$ & 0.00198 & -1.47676 \\
\hline 0.06 & $12.859_{-0.097}^{+0.096}$ & $5.12298 \times 10^{14}$ & $3.23275 \times 10^{14}$ & $2.78676 \times 10^{34}$ & $2.8133 \times 10^{20}$ & 0.00208 & -1.56545 \\
\hline 0.077 & $12.774_{-0.096}^{+0.096}$ & $5.20514 \times 10^{14}$ & $3.30354 \times 10^{14}$ & $2.79651 \times 10^{34}$ & $2.7932 \times 10^{20}$ & 0.00216 & -1.63736 \\
\hline
\end{tabular}

Table 3 Comparative study of lower bound, mass-radius ratio, upper bound, compactness $\left(u=M_{\mathrm{eff}} / R\right)$ and surface red-shift of the star PSR J1416-2230 for different values of $\alpha$ with $\beta=1.1, \gamma=-0.001, a=0.003$, and $b=0.0000053$

\begin{tabular}{lllllll}
\hline$\alpha$ & $\begin{array}{l}\text { predicted } \\
\text { radius } \mathrm{R}(\mathrm{km})\end{array}$ & $\begin{array}{l}\text { Lower bound } \\
\left(\frac{Q^{2}\left(18 R^{2}+Q^{2}\right)}{2 R^{2}\left(12 R^{2}+Q^{2}\right)}\right)\end{array}$ & Mass-radius ratio $\left(\frac{M}{R}\right)$ & $\begin{array}{l}\text { compactness }(u) \\
\left(\frac{M_{\text {eff }}}{R}\right)\end{array}$ & $\begin{array}{l}\text { Upper bound } \\
\left(\frac{2 R^{2}+3 Q^{2}+2 R \sqrt{R^{2}+3 Q^{2}}}{9 R^{2}}\right)\end{array}$ & $\begin{array}{c}\text { Surface redshift }\left(z_{s}\right) \\
0.0375\end{array}$ \\
\hline 0.00 & $13.175_{-0.1}^{+0.1}$ & 0.027105 & 0.220545 & 0.202457 & 0.468251 & 0.29631 \\
0.02 & $13.067_{-0.099}^{+0.099}$ & 0.026747 & 0.222383 & 0.204534 & 0.467940 & 0.300862 \\
0.04 & $12.962_{-0.098}^{+0.097}$ & 0.026513 & 0.224207 & 0.206514 & 0.467738 & 0.305242 \\
0.06 & $12.859_{-0.097}^{+0.096}$ & 0.026384 & 0.225987 & 0.208380 & 0.467625 & 0.309412 \\
0.077 & $12.774_{-0.096}^{+0.096}$ & 0.026356 & 0.227493 & 0.209905 & 0.46760 & 0.31285 \\
\hline
\end{tabular}

decoupled system have been derived corresponding to the total energy momentum tensor $T_{i j}^{\text {tot }}$ by varying the modified action over metric tensor $g^{i j}$. Then decoupled field equations have been divided into two sets of equations using the CGD approach: $e^{-\lambda} \longrightarrow \mu+\alpha f(r)$ and $v(r) \longrightarrow \xi(r)+\alpha h(r)$, where $f(r) \neq 0$ and $h(r) \neq 0$ are called the deformation functions while $\alpha$ is a decoupling constant. The first system represents the Einstein-Maxwell system for isotropic matter distribution $\left(T_{i j}\right)$ which has been solved by using the wellknown spacetime geometry (38), while the second system for $\theta_{i j}$ mainly depends on $f(r)$ and $h(r)$ that has been solved using two different approaches namely the mimic approach and the equation of state approach. To do this, we mimicked the $\rho(r)$ with the $\theta_{0}^{0}(r)$ i.e. $\rho(r)=\theta_{0}^{0}(r)$ that provides the expression for $f(r)$ while we use the equation of state (EOS) approach, in particular $\theta_{1}^{1}=\beta \theta_{0}^{0}+\gamma$, in order to determine $h(r)$. We highlight that due to the presence of the additional $\theta_{i j}$ source in the system, anisotropy arises for the condition $\theta_{2}^{2} \neq \theta_{1}^{1}$ and then the total energy density $\rho^{\text {tot }}$, total radial pressure $p_{r}^{\text {tot }}$ and total tangential pressure $p_{t}^{\text {tot }}$ becomes a combination of their isotropic versions and addition of the $\theta$ components with a coupling constant $\alpha$ as can be seen from the Eq. (19). We also studied the impact of the coupling constant $\alpha$ in this work which is discussed in the latter half of this section. The junction conditions for the decoupled system in 


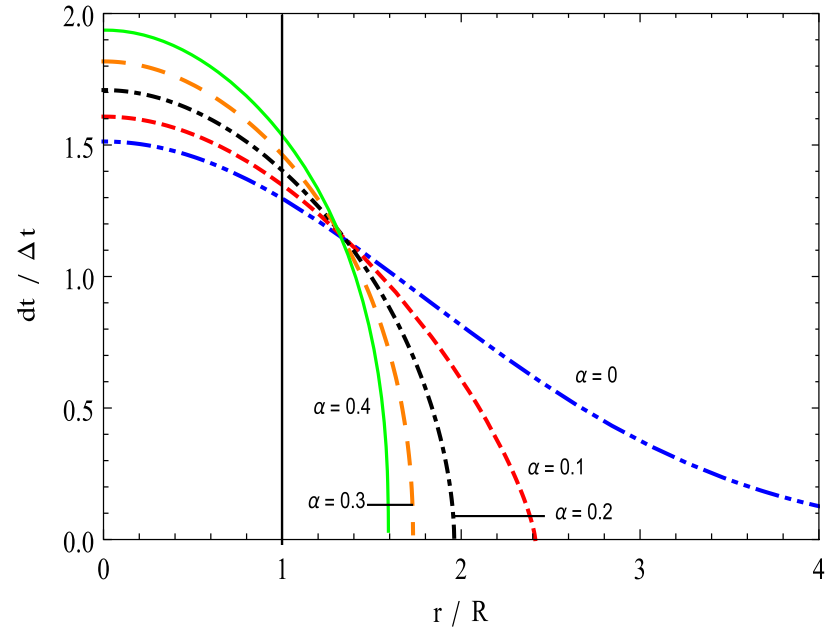

Fig. 15 The variation time dilation $(d t / \Delta t)$ versus radial coordinate $r / R$ for different values of $\alpha$. Here $r / R=1$ is the boundary of the compact objects while $r / R>1$ describes the exterior space-time region

the context of gravitational decoupling have been derived by taking the exterior Ressiner-Nordström spacetime. All the constants involved in the solution are obtained by the matching of the interior $(r<R)$ and exterior $(r>R)$ spacetime at the surface $(r=R)$ together with taking total radial pressure to be zero at $r=R$.

During the analysis of the deformation functions $f(r)$ and $h(r)$, we found that $f(r)$ is zero at the center and as we go towards the boundary, it decreases (Fig. 3 - left panel) which shows that it has an inverse relationship with $e^{\lambda}$. Then, we can say that $e^{\lambda}$ will increase as one moves towards the boundary and $e^{\lambda}=1$ at $r=0$ whereas, $h(r)$ also decreases and is positive as one moves towards the boundary, but it is non-vanishing at the center (Fig. 3 -right panel). But $e^{v}=e^{\xi+\alpha h(r)}$ is increasing monotonically towards the boundary which shows the $\xi$ term grows faster than the term $\alpha h(r)$. The variations of $e^{\lambda}$ and $e^{v}$ within different stellar objects are mentioned in Fig. 4 which satisfy the criteria of realistic self-gravitating objects. On the other hand, Fig. 5 display the variation of radial pressure $\left(p_{r}^{\text {tot }}\right)$ and tangential pressure $\left(p_{t}^{\text {tot }}\right)$, we can see that they decrease monotonically as one moves towards the boundary and attains the maximum value at the center where the radial pressure decreases in a slightly steeper manner. Also, the total energy density $\rho^{\text {tot }}$ is maximum at the center and decreases monotonically towards the boundary which in turn implies that the energy density is vastly concentrated near the center of the compact stellar object, see Fig. 6 (left panel). Furthermore, from the right panel of Fig. 6, it can be seen that the anisotropic factor $\Delta$ is zero at the center (i.e. the matter distribution can be said as perfect fluid at the center) and as one moves towards the surface, the anisotropy increases and becomes maximum near the surface. This implies that the anisotropic force
$\left(F_{a}=2 \Delta / r\right)$ acts radially outwards and this plays an active role in preventing gravitational collapse. Next, we studied the several energy conditions as mentioned in Sect. 6.2, the Null energy condition (NEC), weak energy conditions (WEC), strong energy condition (SEC) together with dominant (DEC) and trace (TEC) energy conditions. Since all first three conditions will automatically satisfy due to positiveness of $p_{r}^{\text {tot }}, p_{t}^{\text {tot }}, \rho^{\text {tot }}$ and $q$, therefore only we plot the Fig. 7 for the dominant and trace energy condition to check their validity inside each object. We found that both DEC and TEC are satisfying within each point of the stellar objects. The variation of the $m / r$ ratio within the objects is shown in Fig. 8. Form this variation, we found that $m / r$ ratio is increasing towards the boundary but is less than the Buchdahal upper bound value $4 / 9$. On the other hand, Lower bound, $M / R$, compactness $M_{\mathrm{eff}} / R$, upper bound and surface redshift $\left(z_{s}\right)$ values are displayed in Table 1 . The surface redshift values are increasing when compactness $\left(M_{\mathrm{eff}} / R\right)$ increases. The calculated values of $z_{s}$ for different object, by considering $\beta=1.1$, $\gamma=-0.001, a=0.0003, b=0.0000053$ and the decoupling constant $\alpha=0.02$, are as follows: (i) $z_{s}=0.3008625$ for PSR J1416-2230, (ii) $z_{s}=0.2757358$ for Vela X-1, (iii) $z_{s}=0.2718340$ for $4 \mathrm{U} 1608-52$, (iv) $z_{s}=0.262529$ for PSR J1903+327, (v) $z_{s}=0.2514419$ for $4 \mathrm{U} 1820-30$, (vi) $z_{s}=0.2398789$ for Cen X-3, (vii) $z_{s}=0.215279$ for EXO 1785-248, (viii) $z_{s}=0.213986$ for LMC X-4, (ix) $z_{s}=0.1808966$ for SMC X-1, (x) $z_{s}=0.161925$ for SAX $\mathrm{J} 1808.4-3658$, (xi) $z_{s}=0.1577812$ for $4 \mathrm{U} 1538-52$ and (xii) $z_{s}=0.155007$ for Her X-1. Moreover, we found that the $M / R$ ratio clearly satisfied both inequalities (74) and (75). Since as $M>M_{\text {eff }}$, then we can infer that $M_{\text {eff }} / R$ will also lie inside the upper and lower bound. The stability analysis and dynamical equilibrium has been also discussed. From Fig. 9, we found that both radial and tangential velocities remain less than the unity which implies that the velocity of sound is lesser than the velocity of light throughout the model that supports the criteria of a realistic model. While studying the stability of the solution based on Herrera and Abreu's criteria, we found from Fig. 10 that the stable zone obtained from the Eq. (78) is maintained throughout the model and all the regions are completely stable in the system. Looking into the hydrostatic equilibrium through generalized TOV equation under extended gravitational decoupling, we found from Fig. 11 that for all different 12 stars, the gravitational force $\left(F_{g}^{\text {tot }}\right)$ is acting strongly inwards and other forces like anisotropic force $\left(F_{a}^{\text {tot }}\right)$, electric force $\left(F_{e}^{\text {tot }}\right)$ and hydrostatic force $\left(F_{h}^{\text {tot }}\right)$ are acting outwards. It is also seen that $F_{g}^{\text {tot }}$, $F_{a}^{\text {tot }}, F_{e}^{\text {tot }}$ are increasing monotonically throughout the model whereas, the $F_{h}^{\text {tot }}$ increases up to $r / R \approx 0.6$ where it attains its maxima and decreases after that. Also, all the forces combine to zero net force, i.e. $F_{h}^{\text {tot }}+F_{g}^{\text {tot }}+F_{a}^{\text {tot }}+F_{e}^{\text {tot }}=0$, from which we can infer that the hydrostatic equilibrium is maintained for each compact object. 


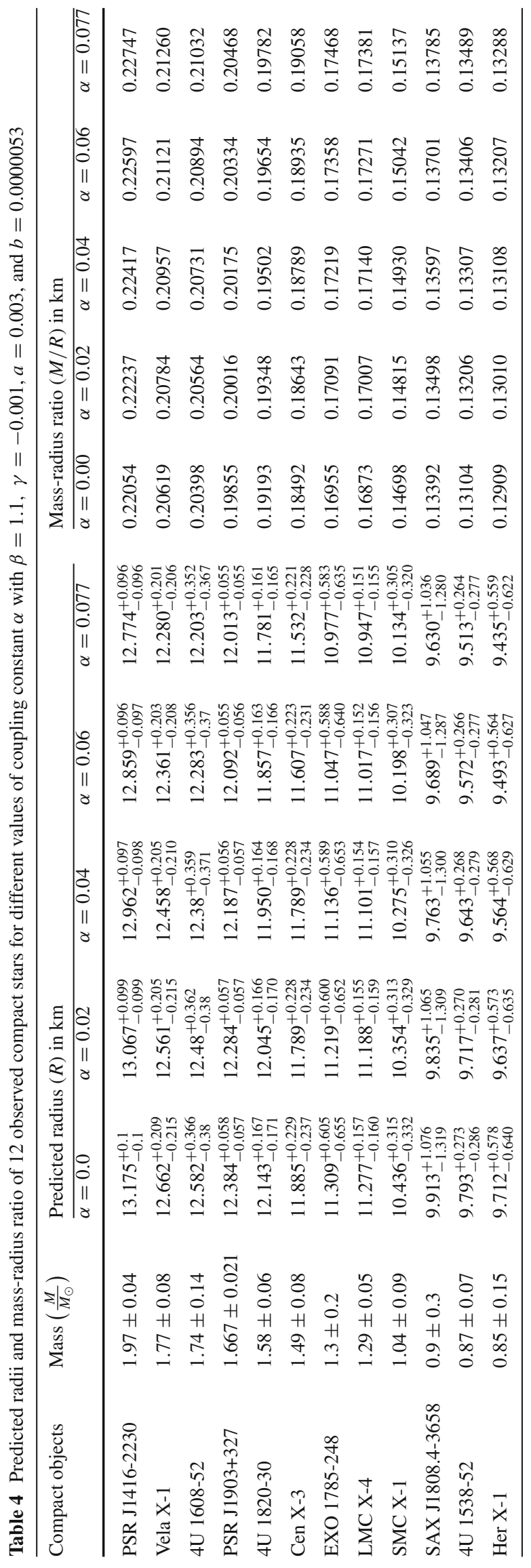


Focusing on the impact of the coupling constant $\alpha$, we plot the Figs. 12, 13 and 14 for particular star PSR J14162230. From Fig. 12, we observe that when $\alpha=0$, the matter distribution is isotropic and when $\alpha$ increases, the two pressure components bifurcate and anisotropy arises into the system and this anisotropy is maximum near the surface. It also shows that if $\alpha$ increases then $p_{\text {anis }}^{\text {tot }}>p_{\text {iso }}^{\text {tot } 8}$ at $r=0$. Also, both central and surface densities are increasing when $\alpha$ increases, in particular as shown in Fig. 13 when $\alpha$ tends $0 \longrightarrow 0.077$, then isotropic matter distribution becomes anisotropic and $\rho_{\text {anis }}^{\text {tot }}>\rho_{\text {iso }}^{\text {tot }}, 9$ which strongly supports the Ruderman [90] suggestion that nuclear matter tends to become anisotropic in nature at very high densities of the order $10^{15} \mathrm{~g} / \mathrm{cm}^{3}$. The numerical values for $p_{c}^{\text {tot }}, \rho_{c}^{\text {tot }}$ and $\rho_{s}^{\text {tot }}$ are displayed in Table 2 for different values of $\alpha$. Also from Fig. 14, it is seen that the electric charge is decreasing while mass-radius ratio increases within the stellar model when $\alpha$ increases. Also, as analyzing Table 3 we can infer that the charge $Q$ on the boundary decreases with the increase of $\alpha$. Also, it can be seen that both $M / R$ and $M_{\text {eff }} / R$ lie between in the lower and upper bounds of each obtained value of $\alpha$. Moreover, both $M / R$ and $M_{\text {eff }} / R$ and also the surface redshift $z_{s}$ increases with $\alpha$. Finally, we also predicted the radius for each obtained stellar object for different values of decoupling constant $\alpha$ together with mass-radius ratio which are displayed in Table 4 . The predicted radii for the objects with mass $\geq 1.5 M_{\odot}$ are lying between $11-13.5 \mathrm{~km}$. Then our predicted radii are strongly compatible with the physical radius for several typical NS equations of state (EOS) obtained by Lattimer and Prakash [91]. Finally, we would like to say that the gravitational decoupling approach plays a vital role to obtain well-behaved realistic compact objects from isotropic to anisotropic matter distributions.

Acknowledgements S. K. Maurya et al. acknowledge that this work is carried out under TRC project-BFP/RGP/CBS/19/099 of the Sultanate of Oman. The authors also acknowledge for continuing support and encouragement from the administration of University of Nizwa.

Data Availability Statement This manuscript has no associated data or the data will not be deposited. [Authors' comment: There is no external data associated with this manuscript.]

Open Access This article is licensed under a Creative Commons Attribution 4.0 International License, which permits use, sharing, adaptation, distribution and reproduction in any medium or format, as long as you give appropriate credit to the original author(s) and the source, provide a link to the Creative Commons licence, and indicate if changes were made. The images or other third party material in this article are included in the article's Creative Commons licence, unless indicated otherwise in a credit line to the material. If material is not included in the article's Creative Commons licence and your intended

$\overline{8 p_{\text {anis }}^{\text {tot }} \text { denotes }}$ the anisotropic pressure when $\alpha \neq 0$ while $p_{\text {iso }}^{\text {tot }}$ is a isotropic pressure corresponding to $\alpha=0$.

$9 \rho_{\text {anis }}^{\text {tot }}$ denotes the anisotropic density corresponding to $\alpha \neq 0$ while $\rho_{\text {iso }}^{\text {tot }}$ is the energy density for isotropic matter distribution when $\alpha=0$. use is not permitted by statutory regulation or exceeds the permitted use, you will need to obtain permission directly from the copyright holder. To view a copy of this licence, visit http://creativecomm ons.org/licenses/by/4.0/.

Funded by $\mathrm{SCOAP}^{3}$.

\section{Appendix}

The values of expressions for the used coefficients as above

$$
\begin{aligned}
& f_{1}=\sqrt{2}\left[6 b^{2}+2 a\left(-a+\sqrt{a^{2}-4 b}\right) B^{2}+b B(-3 a\right. \\
& \left.\left.+3 \sqrt{a^{2}-4 b}+4 B\right)\right], \quad f_{3}=\sqrt{a-\sqrt{\left(a^{2}-4 b\right)}} \\
& f_{2}=\sqrt{2}\left[6 b^{2}-2 a\left(a+\sqrt{a^{2}-4 b}\right) B^{2}+b B(-3 a\right. \\
& \left.\left.-3 \sqrt{a^{2}-4 b}+4 B\right)\right], \quad f_{4}(r)=\sqrt{a+\sqrt{a^{2}-4 b}}, \\
& f_{5}=\sqrt{a^{2}-4 b} \\
& B_{0}(R)=8-3 \alpha \beta-6 a R^{2}-a \alpha \beta R^{2}-4 b R^{4}+b \alpha \beta R^{4} \text {, } \\
& B_{1}(R)=\left[8+6 a R^{2}+4 b R^{4}+\alpha \beta\left(3+a R^{2}-b R^{4}\right)\right]^{2}, \\
& B_{2}(R)=a^{2} R^{2}\left(2+5 \alpha \beta+4 \alpha \gamma R^{2}\right)+2 b R^{2}\left(3+b R^{4}\right) \\
& +\alpha\left[4 \gamma\left(1+b R^{4}\right)^{2}+5 b \beta R^{2}\left(5+b R^{4}\right)\right], \\
& B_{3}(R)=4+4 b R^{4}+\alpha\left[8 \gamma R^{2}\left(1+b R^{4}\right)+5 \beta\left(3+2 b R^{4}\right)\right] \\
& h_{1}(r)=\frac{100\left[\psi_{11}(r)+b\left(8 B-5 A_{1} A_{2}\right)\right] \beta \log \left[a-A_{1}+2 b r^{2}\right]}{A_{1}\left[-b\left(A_{2}\right)^{2}+B \alpha\left(-B \alpha+a A_{2}\right)\right]}, \\
& h_{2}(r)=\frac{100\left[B \psi_{11}(r)-b\left(8 B+5 A_{1} A_{2}\right)\right] \beta \log \left[a+A_{1}+2 b r^{2}\right]}{A_{1}\left[-b\left(A_{2}\right)^{2}+B \alpha\left(-B \alpha+a A_{2}\right)\right]}, \\
& h_{3}(r)=4 B^{2} \alpha^{3 / 2}\left(B \alpha-a A_{2}\right)\left[B \sqrt{\alpha}-5 a \sqrt{\alpha}+A_{3}\right) \gamma-5 b^{2} \\
& \times\left[5 B\left(256-48 \alpha(\beta-13)+25 \alpha^{3} A_{4}+120 \alpha^{2}(3+\beta)\right]-A_{2}\right. \\
& \left.\times\left(25 a \alpha A_{2} A_{4}+20 \sqrt{\alpha} A_{3} A_{4}+25 \alpha^{3 / 2} A_{3}(5 \beta-1)+A_{5}\right)\right] \text {. } \\
& h_{4}(r)=A_{2}\left[25 a \alpha A_{2} A_{4}+20 \sqrt{\alpha} A_{3} A_{4}+25 \alpha^{3 / 2} A_{3}(-1+5 \beta)+A_{5}\right] \text {, } \\
& h_{5}(r)=-25 B^{2} \alpha^{3 / 2}\left(16+\alpha A_{4}\right)-125 a^{2} \alpha^{3 / 2}\left(5 \alpha A_{4}-4(1+\beta)\right) \\
& +4 A_{2}^{2} A_{3} \gamma+5 a \sqrt{\alpha}\left[10 B\left(32-2 \alpha(-19+\beta)+5 \alpha^{2} A_{4}\right)\right) \\
& \left.+20 \sqrt{\alpha} A_{3}(1-3 \beta)-25 \alpha^{3 / 2} A_{3}(-1+5 \beta)+A_{6}\right], \\
& h_{6}(r)=h 3+b B \sqrt{\alpha}\left[h 5+B \sqrt{\alpha}\left(25 \alpha^{3 / 2} A_{3}(-1+5 \alpha)+A_{7}\right]\right. \text {, } \\
& h_{7}(r)=b \alpha^{3 / 2} A_{3}\left[b A_{2}^{2}+B \alpha\left(B \alpha-a A_{2}\right)\right] \text {, } \\
& h_{8}(r)=-4 B^{2} \alpha^{3 / 2}\left(-B \alpha+a A_{2}\right)\left(5 a \sqrt{\alpha}-B \sqrt{\alpha}+A_{3} \gamma+5 b^{2}\right. \\
& \times\left[5 B\left(256-48 \alpha(\beta-13)+25 \alpha^{3} A_{4}+120 \alpha^{2}(3+\beta)\right)\right. \\
& \left.-A_{2}\left(25 a \alpha A_{2} A_{4}-20 \sqrt{\alpha} A_{3} A_{4}-25 \alpha^{3 / 2} A_{3}(5 \beta-1)+A_{5}\right)\right] \text {, } \\
& h_{9}(r)=25 B^{2} \alpha^{3 / 2}\left(16+\alpha A_{4}\right)+125 a^{2} \alpha^{3 / 2}\left(5 \alpha A_{4}\right)-4(1+\beta) \\
& +4 A_{2} A_{3} \gamma-5 a \sqrt{\alpha}\left[1 0 B \left(32-2 \alpha(-19+\beta)+5 \alpha^{2} A_{4}\right.\right. \\
& \left.+20 \sqrt{\alpha} A_{3}(-1+3 \beta)+25 \alpha^{3 / 2} A_{3}(-1+5 \beta)+A_{6}\right] \text {, } \\
& h_{10}(r)=-25 \alpha^{3 / 2} A_{3}(-1+5 \beta)+A_{7} \text {, } \\
& h_{11}(r)=\log \left[5 a \sqrt{\alpha}-B \sqrt{\alpha}-A_{3}+10 b \sqrt{\alpha} r^{2}\right] \text {, } \\
& h_{12}(r)=\log \left[5 a \sqrt{\alpha}-B \sqrt{\alpha}+A_{3}+10 b \sqrt{\alpha} r^{2}\right] \text {, }
\end{aligned}
$$

$$
\begin{aligned}
A_{0}= & 2+5 \alpha, \quad A_{1}=\sqrt{a^{2}-4 b}, \quad A_{2}=4+5 \alpha, \\
A_{3}= & \sqrt{80 b+(5 a+B)^{2} \alpha}, \quad A_{4}=(\beta-1), \quad A_{5}=(128 \gamma \\
& \left.+320 \alpha \gamma+200 \alpha^{2} \gamma\right), \quad A_{6}=\left(-192 \gamma-480 \alpha \gamma-300 \alpha^{2} \gamma\right), \\
A_{7}= & 64 \gamma+320 \alpha \gamma+300 \alpha^{2} \gamma, \quad \psi_{11}(r)=-2 a^{2}-A_{1} B \alpha+a A_{1} A_{0},
\end{aligned}
$$




$$
\begin{aligned}
F_{1}(r) & =\left(6+B r^{2}+2 b r^{2}-B^{2} r^{4}\right), F_{2}(r)=\left(11+2 B r^{2}-B^{2} r^{4}\right), \\
F_{3}(r) & =4 \gamma+25 b \beta r^{2}+8 b \gamma r^{4}, \quad F_{4}(r)=-3+b r^{4}, \\
F_{5}(r) & =\left(5 \beta r^{2}+4 \gamma r^{4}\right), \quad F_{6}(r)=1+b r^{4}, \\
F_{7}(r) & =\left(15-B r^{2}+10 b r^{4}\right) \\
\theta_{11}(r) & =a^{2} F_{5}(r)+a\left[8 \gamma r^{2} F_{6}(r)+\beta F_{7}(r),\right.
\end{aligned}
$$

$$
\begin{aligned}
\theta_{21}(r)= & -64 \gamma-800 b \beta r^{2}+16 \alpha \gamma^{2} r^{2}-256 b \gamma r^{4} \\
& -40 b \alpha \gamma r^{4}+200 b \alpha \beta \gamma r^{4}+80 b^{2} r^{6}+125 b^{2} \alpha r^{6} \\
& +80 b^{2} \beta r^{6}+250 b^{2} \alpha \beta r^{6}+625 b^{2} \alpha \beta^{2} r^{6}+64 b \alpha \\
& \times \gamma^{2} r^{6}-320 b^{2} \gamma r^{8}-40 b^{2} \alpha \gamma r^{8}+440 b^{2} \alpha \beta \gamma r^{8} \\
& +80 b^{3} r^{10}+150 b^{3} \alpha r^{10}-80 b^{3} \beta r^{10}-1000 b^{3} \alpha \beta r^{10} \\
& +250 b^{3} \alpha \beta^{2} r^{10}+96 b^{2} \alpha \gamma^{2} r^{10}-128 b^{3} \gamma r^{12}+40 b^{3} \\
& \times \alpha \gamma r^{12}+280 b^{3} \alpha \beta \gamma r^{12}+25 b^{4} \alpha r^{14}-50 b^{4} \alpha \beta r^{14} \\
& +25 b^{4} \alpha \beta^{2} r^{14}+64 b^{3} \alpha \gamma^{2} r^{14}+40 b^{4} \alpha \gamma r^{16}+40 b^{4} \\
& \times \alpha \beta \gamma r^{16}+16 b^{4} \alpha \gamma^{2} r^{18}-16 B^{3} r^{4}\left(1+b r^{4}\right)^{2}, \\
\theta_{22}(r)= & a^{4} \alpha r^{6}\left(25 \beta^{2}+10 \beta\left(-5+4 \gamma r^{2}\right)+\left(5+4 \gamma r^{2}\right)^{2}\right) \\
& +B^{2} r^{2}\left[16\left(1+b r^{4}\right)\left(3 b r^{4}+5 b^{2} r^{8}-2+\beta\left(3-b r^{4}\right)\right)\right. \\
& +\alpha\left(3+2 b r^{4}-b^{2} r^{8}+4 b \beta r^{4}\left(b r^{4}-11\right)+\beta^{2}\left(b r^{4}\right.\right. \\
& \left.\left.-3)^{2}\right)\right]+2 a^{3} r^{4}\left(4 \left(5+20 B r^{2}-12 \gamma r^{2}+10 B^{2} r^{4}\right.\right. \\
& \left.-8 B \gamma r^{4}-5 \beta\left(1+2 B r^{2}\right)\right)+\alpha\left(50-5 B r^{2}+40 \gamma r^{2}\right. \\
& +50 b r^{4}-4 B \gamma r^{4}+32 \gamma^{2} r^{4}+80 b \gamma r^{6}+32 b \gamma^{2} r^{8} \\
& +\beta^{2}\left(75-5 B r^{2}+50 b r^{4}\right)+\beta\left(10 B r^{2}-175+100 \gamma r^{2}\right. \\
& \left.\left.\left.-100 b r^{4}-4 B \gamma r^{4}+80 b \gamma r^{6}\right)\right)\right)+2 B\left(5 b \alpha \beta^{2} r^{4}(-15\right. \\
& \left.+2 b r^{4}+b^{2} r^{8}\right)-4 r^{2}\left(1+b r^{4}\right)\left(b r^{2}\left(5 \alpha-30 b r^{4}-28\right)\right. \\
& \left.+2 \gamma\left(1+b r^{4}\right)\left(4+b(4+\alpha) r^{4}\right)\right)+\beta\left(-8\left(34 b r^{4}-3\right.\right. \\
& \left.+30 b^{2} r^{8}+5 b^{3} r^{12}\right)+\alpha r^{2}\left(4 \gamma\left(-3+b r^{4}\right)\left(1+b r^{4}\right)^{2}\right. \\
& \left.\left.\left.-5 b r^{2}\left(7-38 b r^{4}+3 b^{2} r^{8}\right)\right)\right)\right),
\end{aligned}
$$

$$
\begin{aligned}
\theta_{23}(r)= & 8\left(5-32 \gamma r^{2}+20 b r^{4}-2 B^{3} r^{6}-40 b \gamma r^{6}+2 B^{2} r^{4}(8\right. \\
& \left.+15 b r^{4}\right)+\beta\left(2 B^{2} r^{4}-5-39 B r^{2}-20 b r^{4}-30 b B r^{6}\right) \\
& \left.+B\left(39 r^{2}-24 \gamma r^{4}+70 b r^{6}-24 b \gamma r^{8}\right)\right)+\alpha\left(75+40 \gamma r^{2}\right. \\
& +350 b r^{4}+B^{2} r^{4}+96 \gamma^{2} r^{4}+200 b \gamma r^{6}+150 b^{2} r^{8} \\
& +192 b \gamma^{2} r^{8}+240 b^{2} \gamma r^{1} 0+96 b^{2} \gamma^{2} r^{12}-4 B r^{2}(10 \\
& \left.+4 \gamma r^{2}+5 b r^{4}+8 b \gamma r^{6}\right)+\beta^{2}\left(B^{2} r^{4}-10 B r^{2}\left(6+b r^{4}\right)\right. \\
& \left.+25\left(9+22 b r^{4}+6 b^{2} r^{8}\right)\right)-2 \beta r^{2}\left(B^{2} r^{2}+B\left(4 \gamma r^{2}(5\right.\right. \\
& \left.\left.+b r^{4}\right)-5\left(14+9 b r^{4}\right)\right)-10\left(-5 b r^{2}\left(13+3 b r^{4}\right)+2 \gamma\right. \\
& \left.\left.\left.\left.\times\left(7+17 b r^{4}+6 b^{2} r^{8}\right)\right)\right)\right)\right)-2 a\left(\alpha \beta ^ { 2 } r ^ { 2 } \left(B^{2} r^{2}\left(b r^{4}-3\right)\right.\right. \\
& \left.-5 B\left(-9-8 b r^{4}+b^{2} r^{8}\right)-25 b r^{2}\left(15+13 b r^{4}+2 b^{2} r^{8}\right)\right) \\
& -r^{2}\left(-16 B^{3}\left(r^{4}+b r^{8}\right)+2 B^{2} r^{2}\left(4+\alpha+64 b r^{4}+60 b^{2} r^{8}\right)\right. \\
& +2\left(16 \alpha \gamma^{2} r^{2}\left(1+b r^{4}\right)^{3}+8 \gamma\left(1+b r^{4}\right)\left(-7-11 b r^{4}\right.\right. \\
& \left.\left.+5 b^{2} \alpha r^{8}\right)+5 b r^{2}\left(6+10 b r^{4}+5 \alpha\left(2+4 b r^{4}+b^{2} r^{8}\right)\right)\right) \\
& -B\left(4\left(-19-97 b r^{4}-80 b^{2} r^{8}+24 \gamma\left(r+b r^{5}\right)^{2}\right)+\alpha(5\right. \\
& \left.\left.\left.\times\left(3+8 b r^{4}+b^{2} r^{8}\right)+4 \gamma\left(r^{2}+6 b r^{6}+5 b^{2} r^{10}\right)\right)\right)\right), \\
\theta_{24}(r)= & \beta\left(B^{2} r^{4}\left(-32+\alpha\left(7+5 b r^{4}\right)\right)-4 B r^{2}\right. \\
& \times\left(-29-105 b r^{4}-30 b^{2} r^{8}+\alpha r^{2}\left(5 b r^{2}\left(12+b r^{4}\right)\right.\right. \\
& \left.\left.+\gamma\left(-7-6 b r^{4}+b^{2} r^{8}\right)\right)\right)-5\left(-4\left(6+5 b r^{4}+5 b^{2} r^{8}\right)\right. \\
& +\alpha r^{2}\left(-5 b r^{2}\left(-7+39 b r^{4}+4 b^{2} r^{8}\right)+4 \gamma\left(3+18 b r^{4}\right.\right. \\
& \left.\left.\left.\left.+19 b^{2} r^{8}+4 b^{3} r^{12}\right)\right)\right)\right) .
\end{aligned}
$$

\section{References}

1. J. M. Lattimer, New Astronomy Reviews 54, 101 (2010)

2. R.H. Fowler, Mon. Not. R. Astron. Soc. 87, 114 (1926)

3. S. Chandrasekhar, Mon. Not. R. Astron. Soc. 91, 456 (1931)

4. S. Chandrasekhar, Mon. Not. R. Astron. Soc. 95, 207 (1935)

5. R. Ruderman, Ann. Rev. Astron. Astrophys. 10, 427 (1972)

6. V. Canuto, S.M. Chitre, Phys. Rev. Lett. 30, 999 (1973)

7. V. Canuto, Annu. Rev. Astron. Astrophys. 12, 167 (1974)

8. V. Canuto, S.M. Chitre, Phys. Rev. D 9, 1587 (1974)

9. V. Canuto, Annu. Rev. Astron. Astrophys. 13, 335 (1975)

10. V. Canuto, J. Lodenquai, Phys. Rev. D 11, 233 (1975)

11. H. Rago, Astrophys. Space Sci. 183, 333 (1991)

12. R.L. Bowers, E.P.T. Liang, Class. Astrophys. J. 188, 657 (1974)

13. L. Herrera, N.O. Santos, Phys. Rep. 286, 53 (1997)

14. B.V. Ivanov, Phys. Rev. D 65, 104011 (2002)

15. F.E. Schunck, E.W. Mielke, Class. Quantum Gravity 20, 301 (2003)

16. M.K. Mak, T. Harko, Proc. R. Soc. A 459, 393 (2003)

17. V.V. Usov, Phys. Rev. D 70, 067301 (2004)

18. V. Varela, F. Rahaman, S. Ray, K. Chakraborty, M. Kalam, Phys. Rev. D 82, 044052 (2010)

19. F. Rahaman, S. Ray, A.K. Jafry, K. Chakraborty, Phys. Rev. D 82, 104055 (2010)

20. F. Rahaman, P.K.F. Kuhfittig, M. Kalam, A.A. Usmani, S. Ray, Class. Quantum Gravity 28, 155021 (2011)

21. F. Rahaman, R. Maulick, A.K. Yadav, S. Ray, R. Sharma, Gen. Relativ. Gravit. 44, 107 (2012)

22. M. Kalam, F. Rahaman, S. Ray, SkM Hossein, I. Karar, J. Naskar, Eur. Phys. J. C 72, 2248 (2012)

23. D. Deb, S.R. Chowdhury, S. Ray, F. Rahaman, Gen. Relativ. Gravit. 50, $112(2018)$

24. D. Shee, F. Rahaman, B.K. Guha, S. Ray, Astrophys. Space Sci. 361, 167 (2016)

25. S.K. Maurya, Y.K. Gupta, S. Ray, D. Deb, Eur. Phys. J. C 76, 693 (2016)

26. S.K. Maurya, D. Deb, S. Ray, P.K.F. Kuhfittig, Int. J. Mod. Phys. D 28, 1950116 (2019)

27. J. Ovalle, Mod. Phys. Lett. A 23, 3247 (2008)

28. J. Ovalle, Gravitation and Astrophysics (ICGA9) (World Scientific, Singapore, 2010), pp. 173-182

29. L. Randall, R. Sundrum, Phys. Rev. Lett. 83, 3370 (1999)

30. L. Randall, R. Sundrum, Phys. Rev. Lett. 83, 4690 (1999)

31. E. Contreras, P. Bargueno, Eur. Phys. J. C 78, 985 (2018)

32. E. Contreras, Eur. Phys. J. C 78, 678 (2018)

33. J. Ovalle, F. Linares, Phys. Rev. D 88, 104026 (2013)

34. J. Ovalle, F. Linares, A. Pasqua, A. Sotomayor, Class. Quantum Gravity 30, 175019 (2013)

35. R. Casadio, J. Ovalle, R. da Rocha, Class. Quantum Gravity 30, 175019 (2014)

36. R. Casadio, J. Ovalle, R. da Rocha, Europhys. Lett. 110, 40003 (2015)

37. R. Casadio, J. Ovalle, R. da Rocha, Class. Quantum Gravity 32, 215020 (2015)

38. J. Ovalle, Phys. Rev. D 95, 104019 (2017)

39. J. Ovalle, R. Casadio, A. Sotomayor, Adv. High Energy Phys. 2017, 9 (2017)

40. J. Ovalle, R. Casadio, R. da Rocha, A. Sotomayor, Eur. Phys. J. C 78, 122 (2018)

41. E. Morales, F. Tello-Ortiz, Eur. Phys. J. C 78, 841 (2018)

42. M. Estrada, F. Tello-Ortiz, Eur. Phys. J. Plus 133, 453 (2018)

43. E. Morales, F. Tello-Ortiz, Eur. Phys. J. C 78, 618 (2018)

44. L. Gabbanelli, A. Rincon, C. Rubio, Eur. Phys. J. C 78, 370 (2018)

45. C. Las Heras, P. Leon, Fortschr. Phys. 66, 1800036 (2018)

46. A.R. Graterol, Eur. Phys. J. Plus 133, 244 (2018)

47. J. Ovalle, A. Sotomayor, Eur. Phys. J. Plus 133, 428 (2018) 
48. L. Gabbanelli, J. Ovalle, A. Sotomayor, Z. Stuchlik, R. Casadio, Eur. Phys. J. C 79, 486 (2019)

49. S.K. Maurya, F. Tello-Ortiz, Eur. Phys. J. C 79, 85 (2019)

50. S. Hensh, Z. Stuchik, Eur. Phys. J. C 79, 834 (2019)

51. E. Contreras, A. Rincon, P. Bargueno, Eur. Phys. J. C 79, 216 (2019)

52. K.N. Singh, S.K. Maurya, M.K. Jasim, Farook Rahaman, Eur. Phys. J. C 79, 851 (2019)

53. E. Contreras, P. Bargueño, Eur. Phys. J. C 78, 558 (2018)

54. E. Contreras, Class. Quantum Gravity 36, 095004 (2019)

55. R. Casadio et al., Eur. Phys. J. C 79, 826 (2019)

56. R. Casadio, J. Ovalle, Phys. Lett. B 715, 251 (2012)

57. A. Rincon et al., Eur. Phys. J. C 79, 873 (2019)

58. A. Rincon et al., Eur. Phys. J. C 80, 490 (2020)

59. S.K. Maurya, L.S.S. Al-Farsi, Eur. Phys. J. Plus 136, 317 (2021)

60. E. Contreras, J. Ovalle, R. Casadio, Phys. Rev. D 103, 044020 (2021)

61. F. Tello-Ortiz, Eur. Phys. J. C 80, 413 (2020)

62. R. da Rocha, Phys. Rev. D 95, 124017 (2017)

63. R. da Rocha, Eur. Phys. J. C 77, 355 (2017)

64. R. da Rocha, Symmetry 12, 508 (2020)

65. K.N. Singh, S.K. Maurya, M.K. Jasim, F. Rahaman, Eur. Phys. J. C 79, 851 (2019)

66. S.K. Maurya, F. Tello-Ortiz, Phys. Dark Universe 27, 100442 (2020)

67. S.K. Maurya, F. Tello-Ortiz, S. Ray, Phys. Dark Universe 31, 100753 (2021)

68. M. Sharif, A. Majid, Astrophys. Space Sci. 365, 42 (2020)

69. M. Sharif, A. Majid, Eur. Phys. J. Plus 135, 558 (2020)

70. M. Sharif, A. Majid, Phys. Dark Universe 30, 100610 (2020)

71. M. Sharif, Saadia Saba, Eur. Phys. J. C 78, 921 (2018)

72. R.T. Cavalcanti, A. Goncalves da Silva, R. da Rocha, Class. Quantum Gravity 33, 215007 (2016)

73. R. Casadio, J. Ovalle, R. da Rocha, Class. Quantum Gravity 32, 215020 (2015)

74. J. Ovalle, Phys. Lett. B 788, 213 (2019)
75. S.K. Maurya, Eur. Phys. J. C 80, 429 (2020)

76. S.K. Maurya, K.N. Singh, B. Dayanandan, Eur. Phys. J. C 80, 448 (2020)

77. W. Israel, Nuovo Cim. B 44, 1 (1966)

78. G. Darmois, Memorial des Sciences Mathematiques (GauthierVillars, Paris, 1927)

79. S.W. Hawking, G.F.R. Ellis, The Large Scale Structure of SpaceTime (Cambridge University Press, Cambridge, 1973)

80. R. Schoen, S.T. Yau, Commun. Math. Phys. 65, 45 (1979)

81. K.D. Olum, Phys. Rev. Lett. 81, 3567 (1998)

82. M. Visser, B. Bassett, S. Liberati, Nucl. Phys. Proc. Suppl. 88, 267 (2000)

83. M. Visser, Phys. Rev. D 46, 2445 (1992)

84. H.A. Buchdahl, Phys. Rev. D 116, 1027 (1959)

85. H. Andreasson, J. Differ. Equ. 245, 2243 (2008)

86. C.G. Bohmer, T. Harko, Gen. Relativ. Gravit. 39, 757 (2007)

87. R.L. Bowers, E.P.T. Liang, Astrophys. J. 188, 657 (1974)

88. L. Herrera, Phys. Lett. A 165, 206 (1992)

89. H. Abreu, H. Hernández, L.A. Núñez, Calss. Quantum Gravity 24, 4631 (2007)

90. R. Ruderman, Rev. Astron. Astrophys. 10, 427 (1972)

91. J.M. Lattimer, M. Prakash, Neutron star structure and the equation of state. ApJ 550, 426 (2001) 